\title{
Preliminary Planning for Mars Sample Return (MSR) Curation Activities in a Sample Receiving Facility (SRF)
}

\author{
Kimberly T. Tait, ${ }^{1}$ Francis M. McCubbin, ${ }^{2}$ Caroline L. Smith, ${ }^{3,4}$ Carl B. Agee, ${ }^{5}$ Henner Busemann, ${ }^{6}$ \\ Barbara Cavalazzi, ${ }^{7}$ Vinciane Debaille, ${ }^{8}$ Aurore Hutzler, ${ }^{9}$ Tomohiro Usui, ${ }^{10}$ Gerhard Kminek, ${ }^{9}$ \\ Michael A. Meyer, ${ }^{11}$ David W. Beaty, ${ }^{12}$ Brandi L. Carrier, ${ }^{12}$ Timothy Haltigin, ${ }^{13}$ Lindsay E. Hays, ${ }^{11}$ \\ Charles S. Cockell, ${ }^{14}$ Daniel P. Glavin, ${ }^{15}$ Monica M. Grady, ${ }^{16}$ Ernst Hauber, ${ }^{17}$ Bernard Marty, ${ }^{18}$ Lisa M. Pratt, ${ }^{19}$ \\ Aaron B. Regberg, ${ }^{2}$ Alvin L. Smith, ${ }^{12}$ Roger E. Summons, ${ }^{20}$ Timothy D. Swindle, ${ }^{21}$ Nicholas J. Tosca, ${ }^{22}$ \\ Arya Udry, ${ }^{23}$ Michael A. Velbel, ${ }^{24,25}$ Meenakshi Wadhwa, ${ }^{12,26}$ Frances Westall, ${ }^{27}$ and Maria-Paz Zorzano ${ }^{28,29}$
}

${ }^{1}$ Royal Ontario Museum, Department of Natural History, Toronto, Ontario, Canada.

${ }^{2}$ NASA Johnson Space Center, Astromaterials Research and Exploration Science Division, Houston, Texas, USA.

${ }^{3}$ Natural History Museum, Department of Earth Sciences, London, UK.

${ }^{4}$ University of Glasgow, School of Geographical and Earth Sciences, Glasgow, UK.

${ }^{5}$ University of New Mexico, Institute of Meteoritics, Albuquerque, New Mexico, USA.

${ }^{6}$ ETH Zürich, Institute of Geochemistry and Petrology, Zürich, Switzerland.

${ }^{7}$ Università di Bologna, Dipartimento di Scienze Biologiche, Geologiche e Ambientali, Bologna, Italy.

${ }^{8}$ Université Libre de Bruxelles, Bruxelles, Belgium.

${ }^{9}$ European Space Agency, Noordwijk, The Netherlands.

${ }^{10}$ Japan Aerospace Exploration Agency (JAXA), Institute of Space and Astronautical Science (ISAS), Chofu, Tokyo, Japan.

${ }^{11}$ NASA Headquarters, Mars Sample Return Program, Washington, DC, USA.

${ }^{12}$ Jet Propulsion Laboratory, California Institute of Technology, Pasadena, California, USA.

${ }^{13}$ Canadian Space Agency, Saint-Hubert, Quebec, Canada.

${ }_{15}^{14}$ University of Edinburgh, Centre for Astrobiology, School of Physics \& Astronomy, Edinburgh, UK.

${ }_{15}^{15}$ NASA Goddard Space Flight Center, Solar System Exploration Division, Greenbelt, Maryland, USA

${ }^{16}$ The Open University, Milton Keynes, Buckinghamshire, UK.

${ }^{17}$ German Aerospace Center (DLR), Institute of Planetary Research, Berlin, Germany.

${ }^{18}$ Université de Lorraine, CNRS, CRPG, Nancy, France.

${ }^{19}$ Indiana University Bloomington, Earth and Atmospheric Sciences, Bloomington, Indiana, USA.

${ }^{20}$ Massachusetts Institute of Technology, Earth, Atmospheric and Planetary Sciences, Cambridge, Massachusetts, USA.

${ }^{21}$ University of Arizona, Lunar and Planetary Laboratory, Tucson, Arizona, USA.

${ }^{22}$ University of Cambridge, Department of Earth Sciences, Cambridge, UK.

${ }^{23}$ University of Nevada Las Vegas, Las Vegas, Nevada, USA.

${ }^{24}$ Michigan State University, Earth and Environmental Sciences, East Lansing, Michigan, USA.

${ }^{25}$ Smithsonian Institution, Department of Mineral Sciences, National Museum of Natural History, Washington DC, USA.

${ }^{26}$ Arizona State University, Tempe, Arizona, USA.

${ }^{27}$ Centre National de la Recherche Scientifique (CNRS), Centre de Biophysique Moléculaire, Orléans, France.

${ }^{28}$ Centro de Astrobiologia, (CSIC-INTA), Torrejon de Ardoz, Spain.

${ }^{29}$ University of Aberdeen, Department of Planetary Sciences, School of Geosciences, King's College, Aberdeen, UK.

This paper was written by the MSR Science Planning Group 2 (MSPG2) working under a Terms of Reference from NASA and ESA.

(C) Kimberly T. Tait et al., 2021; Published by Mary Ann Liebert, Inc. This Open Access article is distributed under the terms of the Creative Commons License (http://creativecommons.org/licenses/by/4.0), which permits unrestricted use, distribution, and reproduction in any medium, provided the original work is properly cited. 


\section{Table of Contents}

Abstract

Executive Summary

List of Findings

1. Introduction

1.1. Key assumptions

1.2. High-level guiding principles

1.3. Importance of curators and scientific investigators working together

1.4. Goals of initial sample characterization in the Sample Receiving Facility

2. General Attributes of the Sample Receiving Facility and the Samples

2.1. Temperature of the samples at landing, during transport, and at the Sample Receiving Facility

2.1.1. Temperature of the samples during transport

2.1.2. Temperature of the samples within the SRF

2.2. Sample tubes

2.3. Hardware de-integration

2.4. Isolator details

2.4.1. Pristine isolators

2.4.2. Non-pristine sample containers

2.4.3. Cleaning, sterilizing, and monitoring

2.4.4. Gas to interact with the samples

2.4.5. Approved materials for contact with the Mars returned samples

2.5. Overview of analytical instruments for curation

3. Pre-Basic Characterization (Pre-BC) Activities

3.1. Envisioned processes and workflows

3.2. Workspace configuration

3.2.1. Sample tube dust removal

3.2.2. Sample seal integrity

3.2.3. Individual sample tube isolation containers

3.3. Pre-Basic Characterization Summary

4. Basic Characterization (BC)

4.1. Envisioned processes and workflows of Basic Characterization

4.2. Number of isolators and workspace required

4.3. Headspace gas collection

4.4. Opening of the sample tubes

4.5. Basic Characterization Summary

5. Preliminary Examination (PE)

5.1. Envisioned processes and workflows

5.2. Sample catalog

5.3. Preliminary examination summary

6. Sample Processing

6.1. Microparticle processing

6.2. Macrosample processing

7. Timing and Organizational Considerations

8. Future Work and Conclusions

8.1. Future work

8.2. Conclusions

Acknowledgments

Disclosure Statement

References

Acronyms Used

\section{Abstract}

The Mars Sample Return Planning Group 2 (MSPG2) was tasked with identifying the steps that encompass all the curation activities that would happen within the MSR Sample Receiving Facility (SRF) and any anticipated curation-related requirements. An area of specific interest is the necessary analytical instrumentation. The SRF would be a Biosafety Level-4 facility where the returned MSR flight hardware would be opened, the sample tubes accessed, and the martian sample material extracted from the tubes. Characterization of the essential attributes of 
each sample would be required to provide enough information to prepare a sample catalog used in guiding the preparation of sample-related proposals by the world's research community and informing decisions by the sample allocation committee. The sample catalog would be populated with data and information generated during all phases of activity, including data derived concurrent with Mars 2020 sample-collecting rover activity, sample transport to Earth, and initial sample characterization within the SRF. We conclude that initial sample characterization can best be planned as a set of three sequential phases, which we have called Pre-Basic Characterization (Pre-BC), Basic Characterization (BC), and Preliminary Examination (PE), each of which requires a certain amount of instrumentation. Data on specific samples and subsamples obtained during sample safety assessments and time-sensitive scientific investigations would also be added to the catalog. There are several areas where future work would be beneficial to prepare for the receipt of samples, which would include the design of a sample tube isolation chamber and a strategy for opening the sample tubes and removing dust from the tube exteriors.

\section{Executive Summary}

All material collected from Mars (gases, dust, rock, regolith) would need to be carefully handled, stored, and analyzed following Earth return to minimize the alteration or contamination that could occur on Earth and maximize the scientific information that can be attained from the samples now and into the future. A Sample Receiving Facility (SRF) is where the Earth Entry System (EES) would be opened and the sample tubes opened and processed after they land on Earth. Samples should be accessible for research in biocontainment for time-sensitive studies and eventually, when deemed safe for release after sterilization or biohazard assessment, should be transferred out of biocontainment for allocation to scientific investigators in outside laboratories. There are two main mechanisms for allocation of samples outside the SRF: 1) Wait until the implementation of the Sample Safety Assessment Protocol (Planetary Protection) results concludes that the samples are non-hazardous, 2) Render splits of the samples non-hazardous by means of sterilization. To make these samples accessible, a series of observations and analytical measurements need to be completed to produce a sample catalog for the scientific community. Specialist members of the Mars Sample Return Planning Group Phase 2 (MSPG2), referred to here as the Curation Focus Group, have identified four curation goals that encompass all of the activities within the SRF:

1. Documentation of the state of the sample tubes and their contents prior to opening,

2. Inventory and tracking of the mass of each sample,

3. Preliminary assessment of lithology and any macroscopic forms of heterogeneity (on all the samples, noninvasive, in pristine isolators),

4. Sufficient characterization of the essential attributes of each sample to prepare a sample catalog and respond to requests by the sample allocation committee (partial samples, invasive, outside of pristine isolators).

The sample catalog will provide data for the scientific community to make informed requests for samples for scientific investigations and for the approval of allocations of appropriate samples to satisfy these requests. The sample catalog would be populated with data and information generated during all phases of activity, including data derived from the landed Mars 2020 mission, during sample collection and transport to Earth, and reception within the Sample Receiving Facility. Data on specific samples and subsamples would also be generated during curation activ- ities carried out within the Sample Receiving Facility and during sample safety assessments, time-sensitive studies, and a series of initial sample characterization steps we refer to as Pre-Basic Characterization (Pre-BC), Basic Characterization (BC), and Preliminary Examination (PE) phases. A significant portion of the Curation Focus Group's efforts was to determine which instrumentation would be required to produce a sample catalog for the scientific community and how and when certain instrumentation should be used. The goal is to provide enough information for the PIs to request material for their studies but to avoid facilitating studies that target scientific research that is better left to peer-reviewed competitive processes.

We reviewed the proposed scientific objectives of the International MSR Objectives and Samples Team (iMOST) (Beaty et al., 2019) to make sure that the instrumentation suggested is sufficient to cover these key science planning questions (Table 1; Section S-6). It was determined that for Pre-Basic Characterization, two instruments are required, a Magnetometer (see Section S-1.1) and an X-ray Computed Tomography scanner (XCT see Section S-1.2). For Basic Characterization, there are four instruments that are considered necessary, which are analytical balance(s) (see Section S-2.1), binocular microscopes (see Section S-2.2), and multispectral imaging and hyperspectral scanning systems (see Section S-2.3). Then in Preliminary Examination, there is a set of instruments that should be available for generating more detailed information for the sample catalog. These are a Variable Pressure-Field Emission Scanning Electron Microscope (VP-FE-SEM see Section S-3.1), Confocal Raman spectrometer (see Section S-3.2), Deep UV Fluorescence (see Section S-3.3), a Fourier Transform Infrared Spectrometer (see Section S-3.4), a Micro X-ray Diffractometer (see Section S-3.5), X-ray Fluorescence Spectrometer (see Section S-3.6), and Petrographic and Stereo Microscope (see Section S-3.7). All instruments are summarized in Table 1. Finally, our Curation Focus Group has outlined several specific findings for sample curation within the SRF to complete the sample catalog prior to sample distribution and made several recommendations for future work (summarized in Section 8.1) to build upon the efforts that generated this report.

\section{List of Findings}

MAJOR FINDING C-1: The initial sample characterization in the Sample Receiving Facility of the MSR samples can be broken down into three stages for 


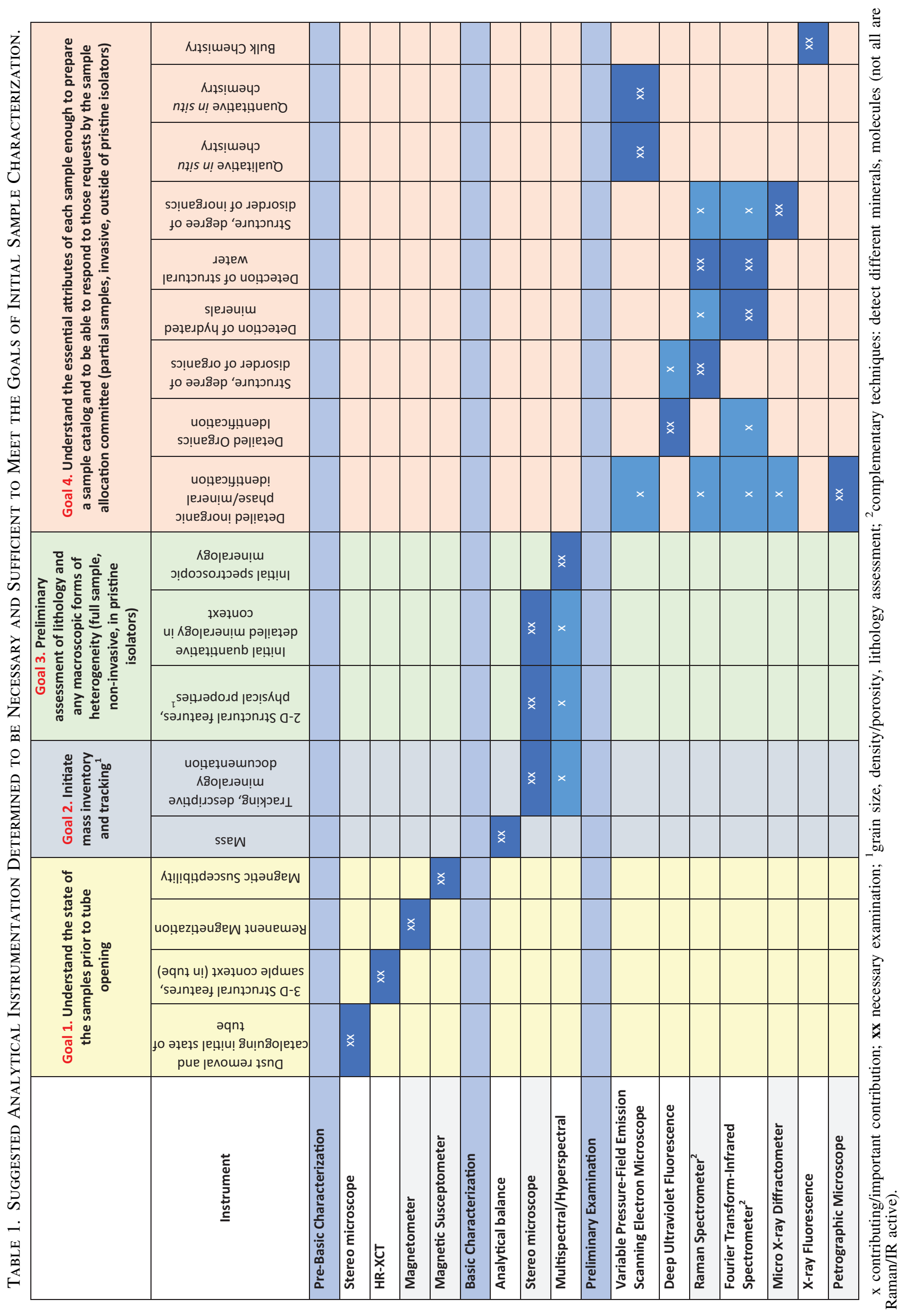


simplicity as follows: Pre-Basic Characterization (PreBC), Basic Characterization (BC), and Preliminary Examination (PE). While the whole collection would be assessed through Pre-BC and $\mathrm{BC}$, only subsets of samples would be used during the PE phase.

FINDING C-2: Immediately after Earth landing, the spacecraft would be recovered and placed in a container designed to control and stabilize its physical conditions. The optimum temperature $\left(\mathrm{T}_{\text {optimum }}\right)$ of the sample tubes during transport to the Sample Receiving Facility (SRF) should be the same as the operating temperature of the SRF to avoid unnecessary temperature shock.

FINDING C-3: The Sample Receiving Facility (SRF) should operate at room temperature $\left(\sim 15-25^{\circ} \mathrm{C}\right)$, and the samples should be held at this temperature through all steps of initial sample characterization, with the option for cold storage of subsamples available in the SRF when needed.

MAJOR FINDING C-4: Measurements on all the sample tubes before they are opened are essential to conduct as the samples could be compromised upon opening of the tubes. This step is called Pre-Basic Characterization (Pre-BC). These are measurements that would inform how the tubes are opened, processed, and subsampled during Basic Characterization (BC).

MAJOR FINDING C-5: Careful collection and storage of the serendipitous dust on the outside of the sample tubes is a critical step in the curation process in the Sample Receiving Facility. The dust collected is a valuable resource to the scientific community.

MAJOR FINDING C-6: Careful collection and storage of the unaltered and unfractionated headspace gas collected from the sample tubes is a critical step in the curation process in the Sample Receiving Facility. The gas collected is a valuable resource to the scientific community.

FINDING C-7: To minimize the interaction of Earth atmospheric gases and gases that are in the sealed sample tubes, once the dust is removed from the exterior of the sample tubes, they should be placed into individual sample tube isolation chambers (STIC) as quickly as possible.

FINDING C-8: There are compelling reasons to perform penetrative $3 \mathrm{D}$ imaging prior to opening the sample tubes. A laboratory-based X-ray Computed Tomography scanner is the best technique to use and the least damaging to organics of the penetrative imaging options considered.

MAJOR FINDING C-9: Measurements on all the samples once the sample tubes are opened within the pristine isolators are essential to make initial macroscopic observations such as weighing, photographing, and optical observations. The first step to this stage is removal and collection of the headspace gas, which then starts the clock for time-sensitive measurements. This step is called Basic Characterization (BC).

FINDING C-10: To avoid cross contamination between samples, it is recommended that, for processing through the isolators, the samples are organized into groups that have like properties. Given what we know about the geology of Jezero Crater, a reasonable starting assumption is five such groups.

MAJOR FINDING C-11: Assuming that sample processing rates are reasonable and the samples are organized into five sets for cross contamination avoidance pur- poses, at least twelve pristine isolators are required to perform Basic Characterization on the MSR samples. This total would increase by two for each additional distinct processing environment.

MAJOR FINDING C-12: More advanced measurements on subsamples, beyond those included in $\mathrm{BC}$, are essential for the allocation of material to the scientific community for investigation, including some measurements that can make irreversible changes to the samples. These types of measurements take place during Preliminary Examination (PE).

FINDING C-13: The output of the initial sample characterization, and a key function of the curation activities within the Sample Receiving Facility, is to produce a sample catalog that would provide relevant information on the samples' physical and mineralogical/chemical characteristics (derived from the Pre-Basic Characterization, Basic Characterization, and Preliminary Examination investigations), sample safety assessments, timesensitive studies, and information derived from mission operations to enable allocation of the most appropriate materials to the scientific community.

FINDING C-14: A staffing model for curation activities, including technical support and informatics/ documentation support, should be developed (as part of ongoing Sample Receiving Facility development) to ensure that the Sample Receiving Facility is staffed appropriately to support sample curation activities.

FINDING C-15: To reduce the risk of catastrophic loss of samples curated in a single facility up to, and including, decadal timescales, the sample collection should be split-once it is possible to do so-and housed in more than one location for the purpose of maximizing the longterm safety of the collection.

\section{Introduction}

$\mathbf{T}$ he Mars Sample Return Science Planning Group Phase 2 (MSPG2) Curation Focus Group (FG) - co-chaired by Francis McCubbin, NASA Johnson Space Center, USA; Caroline Smith, Natural History Museum, UK; and Kim Tait, Royal Ontario Museum, Canada-was tasked with identifying the Mars Sample Return (MSR) curation requirements that would guide the design of the Sample Receiving Facility (SRF). The SRF would include instruments and laboratory space needed to characterize and examine the Mars samples upon their arrival on Earth. Descriptions of the Mars samples documented in the SRF would be published as a sample catalog for the Mars sample science community for both objective-driven and opportunity-driven investigations. Objective-driven investigations are sample analyses and studies performed to address MSR Campaign L1 (Level 1) science objectives in support of Campaign success criteria (Haltigin et al., 2021). These investigations should be competitively selected up to 7 years in advance of samples being returned to Earth, with initial investigations nominally ending 2 years after return. Selected investigation teams would collectively form the MSR Sample Science Team (MSST) (Haltigin et al., 2021). Opportunity-driven investigations are sample analyses and studies that are not explicitly conducted to address MSR Campaign L1 objectives and success criteria. Investigators would apply for sample access and, if deemed 
meritorious through a TBD selection process, would be provided a sample allocation to conduct their research. These investigations would commence after samples have been returned to Earth and continue indefinitely into the future. Opportunity-driven selected investigators and their teams will not include members of the MSST.

\subsection{Key assumptions}

- A biological containment and curation facility equivalent to a Bio-Safety Level 4 (BSL-4) Sample Receiving Facility (SRF) would be located in the United States. This facility would be responsible for the initial receipt of all returned flight hardware, including the samples. Within the SRF, the Earth Entry System (EES) would be opened and the samples extracted. This primary SRF would provide sample biocontainment until such time as the samples are transferred (under biocontainment) to another equivalently rated facility or are deemed safe for use in laboratories without biocontainment (from MSPG2 Terms of Reference).

- The Mars returned samples will be in demand by scientists worldwide. Samples should be accessible for research in biocontainment for time-sensitive studies, and eventually, if safe for release, samples would be transferred out of biocontainment for allocation to PIs and outside laboratories.

- The number of returned sample tubes would likely be in the range of 10-30, and they would be put in priority order for tube opening and sample processing during the return flight. The returned sample mass should be in the range of $\sim 300-500 \mathrm{~g}$, but the final mass depends on numerous factors such as sample type and properties and the number of tubes returned.

- The types of samples in the tubes would be varied and determined by the M2020 team, but would likely include martian gases, dust, rock, regolith that would require different curation pathways for characterization.

- A sample catalog should be produced in a timely manner, so that science investigators can start selecting samples for their investigations. There should be the ability to release information as it becomes available.

\subsection{High-level guiding principles}

- All material (gases, dust, rock, regolith) returned from Mars will be incredibly precious and therefore should be stored, handled, and allocated in such a way as to maximize science return.

- Documentation of the samples and their environmental histories should be provided to researchers to guide sample selection and requests.

- The array of Returnable Sample Tube Assembly (RSTA), here after called sample tubes for simplicity, (no matter what the number is) is regarded as a single sample set (as they will be from a single mission).

- Some fraction of the Mars returned samples should be preserved for posterity and future studies.

- Documentation of the Mars returned samples, as determined in Pre-Basic Characterization (Pre-BC), Basic Characterization $(\mathrm{BC})$, and Preliminary Examination would be crucial to the MSR stakeholders and should be made available to them in a timely fashion.

- MSR curation should start before samples are returned to Earth. MSR Curators should work with the MSR campaign missions (including M2020) and science stakeholders (the Campaign Science Group (CSG), for example) to plan curatorial operations and workflows within the SRF.

- MSR Curation should involve a wide range of scientific and/or technical experts whose priority will be to maintain the scientific integrity of the samples within the curation environment in both the SRF and longterm curation facility(ies). These experts should work closely with stakeholders to maximize the scientific value and utility of the samples.

- Because prompt scientific results from the MSR samples are important to the MSR stakeholders, processing samples to the point that they can be made available to scientific investigation teams beyond the SRF should be done as expeditiously as possible.

- Curators within the SRF should be included in the process for initial sample receiving from the EES. Curation within the SRF should include initial sample receiving from the EES and processing samples for allocations to researchers potentially working within the SRF, for example, as part of the SSAP or on timesensitive studies.

\subsection{Importance of curators and scientific investigators working together}

Scientific investigators and curators have been working together with engineers since the beginning of the Mars Sample Return campaign to define requirements, ensure that the science goals of the mission are met, and collect necessary contamination knowledge data. The Curation FG strongly recommends that this synergetic work continues after the samples are back on Earth. Analytical activities, whether under the remit of curation (initial sample characterization), research (time-sensitive or sterilization-sensitive science, Tosca et al., 2021; Velbel et al., 2021), or safety assessment, should not be done sequentially, but should build upon each other to optimize the sample mass that is used. The Curation FG reiterates that curation activities aim to characterize the samples to enable meaningful research to be done while minimizing sample loss. However, curation should keep analyses minimal so that serendipitous discoveries occur during research investigations. In practice, we recommend that a research advisory group is appointed to oversee the cataloguing work and that curators are consulted for research and safety phases. We consider the sample catalog a living document that incorporates needed data over time no matter the origin of the data.

To be completely devoid of conflict of interest, curators should not lead objective-driven scientific research on the collection under their care, but the curators can serve in a supporting role on objective-driven scientific investigations where their knowledge and expertise are relevant. The guidelines for such participation should be clearly outlined in a "Rules of the Road" document or equivalent. A preplanned sample management plan should be devised to ensure efficiency in the SRF. 


\subsection{Goals of initial sample characterization in the Sample Receiving Facility}

Curation in the SRF would include activities necessary to complete the sample catalog. This initial sample characterization phase, divided into three steps of Pre-BC, BC, and PE, would be implemented with a set of required instruments. Table 1 (Section 2.5) is a traceability matrix that links the proposed required instruments with four main goals that aim to achieve the robust sample catalog needed to support sample allocation for subsequent activities.

KEY DEFINITION: Sample Catalog: All relevant information on the samples' physical and mineralogical/ chemical characteristics (derived from the Pre-Basic Characterization, Basic Characterization, and Preliminary Examination investigations), sample safety assessments, time-sensitive studies, and information derived from mission operations to enable allocation of the most appropriate materials to the scientific community will be compiled in a single document termed the sample catalog.

Goal 1. Document the state of the Orbiting Sample container (OS), sample tubes, and contents prior to opening. This goal would be pursued in the Pre-BC phase and precede all other activities in SRF Curation. Table 1 shows that this would be accomplished through five main activities as follows: 1) During the EES disassembly and extraction, measure the headspace gas pressure and composition between Primary Containment Vessels and Secondary Containment Vessels (PCV and SCV) and OS to evaluate the possibility of tube seal failure (e.g., detection of elevated $\mathrm{CO}_{2}$ gas inside the PCV) even before the samples are removed from the OS; 2) identify any dust in the OS interior and on the tube exterior and remove it to avoid contamination of the sample tube contents and preserve the removed dust for subsequent analysis; 3) verify tube seal integrity which would be crucial to understanding the degree of pristineness of the sample contents (in regards to timesensitive measurements) and whether any headspace gases have escaped; 4) characterize the 3-D structure of the sample tube contents; 5) detect any remnant magnetization of the undisturbed sample contents. These activities would be performed with the four instruments listed under Pre-BC in Table 1.

Goal 2. Sample Mass Inventory and Tracking. This goal would be to create an inventory of the Mars returned samples so that every measurable amount of sample can be tracked and accounted for during Pre-BC, BC, and PE in the SRF, as well as when samples would be allocated and shipped to PIs and outside laboratories, post-PE, and ultimately returned to Curation. The inventory should consist of sample masses with accompanying images that document sample dimensions and physical appearance, and a unique identifying sample number. The inventory should be linked to corresponding documentation of the sample collection process on the surface of Mars and ultimately to the sample catalog (Goal 4 below). Sample mass inventory implementation in the SRF would be carried out with the analytical balances and microscopy equipped with digital cameras listed under BC in Table 1. Multispectral/hyperspectral imaging techniques carried out in BC would also contribute to, and further enhance, the quality of the sample inventory documentation.

Goal 3: Initial Assessment of samples. This goal is entirely part of $\mathrm{BC}$ and aims at recording basic lithologic descriptions including noteworthy macroscopic features such as degree of homogeneity, grain size, color, presence or absence of layering, and other physical attributes. This activity would be limited by the requirement that it is noninvasive, carried out prior to sample subdivision, and with the samples contained in pristine isolators. The results from a preliminary assessment within BC would guide subsequent sample handling and examination activities in PE. Instruments that would be used to achieve the goal of initial assessment are listed under BC in Table 1.

Goal 4: Detailed sample characterization to produce a robust sample catalog. The activities associated with meeting this goal during PE require more advanced measurements on the Mars returned samples in the SRF, which, when combined with the deliverables from meeting Goals 13 , would be sufficient to create and publish a sample catalog guiding curation allocations to the scientific community. The data documented in the sample catalog would include sufficient detail and depth for PIs and science teams to make well-informed decisions on allocation requests for targeted research projects. Table 1 lists nine essential attributes that would be determined for the Mars returned samples through PE. These include first order attributes such as mineralogy, abundance and nature of any organics present, water content, and chemical composition. When required, PE would include removal of a sample from pristine isolators, sample subdivision, and invasive or destructive sample preparation and analysis. In all cases, PE should follow the guiding principle of striving to preserve the integrity of pristine samples. To maximize the amount of sample that remains pristine, the amount of sample used for PE should be the minimum amount that is sufficient to produce the sample catalog. Instruments required to determine the essential attributes of the Mars returned samples are listed under PE in table 1 .

MAJOR FINDING C-1: The initial sample characterization in the Sample Receiving Facility of the MSR samples can be broken down into three stages for simplicity as follows: Pre-Basic Characterization (PreBC), Basic Characterization (BC), and Preliminary Examination (PE). While the whole collection would be assessed through Pre-BC and BC, only subsets of samples would be used during the $\mathrm{PE}$ phase.

\section{General Attributes of the Sample Receiving Facility and the Samples}

A sample receiving facility (SRF) would be where the EES is opened, and the sample tubes would be opened and processed after they land on Earth. NASA's Office of Planetary Protection has deemed that samples from Mars are categorized as "restricted" and need Biosafety Level 4 (BSL-4) containment until deemed safe for release after 
biohazard assessment or sterilization. One element of the Committee on Space Research (COSPAR) activities is to maintain a planetary protection policy for spacefaring nations, both as an international standard to avoid organic and biological contamination in the exploration and use of space, and to guide compliance with the Outer Space Treaty (OST). The country where the samples land would be responsible for developing procedures and policies for protecting Earth from possible life forms that may be returned in these samples.

The SRF should have an array of safety precautions to ensure protection of Earth from the Mars samples and protection of the Mars returned samples from Earth contamination. Physical access to the BSL-4 facility should be limited; there should be significant training and security checks to access the SRF, which could take a significant amount of time. Ideally there would be workspaces or meeting space adjacent to the BSL-4 restricted area to facilitate direct communication between the Curation staff, the Planetary Protection team, and the selected science investigators for the initial objective-driven PI teams. The option for remote access to the facility would be important as well, for science investigators unable to travel to the facility.

\subsection{Temperature of the samples at landing, during transport, and at the Sample Receiving Facility}

Mars has a thin atmosphere, and surface temperatures fluctuate wildly on both diurnal and annual cycles. Material on the surface of Mars will be exposed to temperatures averaging approximately $-60^{\circ} \mathrm{C}$ and ranging from approximately $-125^{\circ} \mathrm{C}$ to $20^{\circ} \mathrm{C}$ for extended periods prior to collection (iMOST, 2018; Beaty et al, 2019). Ideally, we do not want the different components of the sample reacting with each other at a temperature significantly higher than they experienced under natural martian conditions. This can lead to issues such as the release of volatiles through dehydration and decarbonation reactions (and also the inverse hydration/ carbonation reactions), degradation of organic compounds through chemical oxidation, reactions within the gas phase inside the tube, diffusion-related processes such as isotopic exchange, and reactions with the tube walls. How can we define the optimum temperature $\left(\mathrm{T}_{\text {optimum }}\right)$ for the samples after they land on Earth? $\mathrm{T}_{\text {optimum }}$ could be stabilized (and monitored) at one of the following ranges:

- terrestrial ambient temperature $\left(20^{\circ} \mathrm{C}\right.$ to $\left.25^{\circ} \mathrm{C}\right)$

- room temperature $\left(15^{\circ} \mathrm{C}\right.$ to $\left.25^{\circ} \mathrm{C}\right)$

- martian ambient $\mathrm{T}\left(-125^{\circ} \mathrm{C}\right.$ to $\left.20^{\circ} \mathrm{C}\right)$

- refrigerated storage $\left(2\right.$ to $\left.5^{\circ} \mathrm{C}\right)$

- frozen storage $\left(<-20^{\circ} \mathrm{C}\right)$

- ultra-low freezer storage $\left(-80^{\circ} \mathrm{C}\right)$

- cryogenic freezer storage $\left(-150^{\circ} \mathrm{C}\right.$ to $\left.-190^{\circ} \mathrm{C}\right)$

There should be one temperature range selected and maintained throughout all stages of sample handling, and where necessary, subsets of material can be stored at a different temperature. Laboratory experiments on meteorites and other terrestrial rocks to test the alteration effects induced by temperature cycling (Libourel et al., 2021) have shown that fluctuations of $\mathrm{T}$ are more detrimental/destructive for the integrity of samples than maintaining at a constant temperature within a specific temperature range.
2.1.1. Temperature of the samples during transport. On the surface of Mars at Jezero Crater, it is known that the samples will be at an average temperature of $-20^{\circ} \mathrm{C}$ (Modelling by Redmond and Bhandari, Sept. 2015, on behalf of Mars 2020 Project, JPL, pers. comm. Brandi Carrier), so one option would be that samples should be immediately placed at $-20^{\circ} \mathrm{C}$ at the EEV recovery site. In actuality, the martian samples would have been through multiple temperature cycles on the martian surface, and the samples would also have been through at least 2-3 temperature cycles before the landing (MAV launch, brazing of the PCV, and Earth entry) based on the current MSR Program architecture. Especially upon entering Earth's atmosphere and landing, the entirety of the spacecraft may reach high temperatures and would have an extremely hot heat shield. This high temperature could influence the samples and affect their integrity. MSR currently has a preliminary maximum temperature requirement of $+30^{\circ} \mathrm{C}$ for the sample tubes through landing and a requirement to provide a temperature history model of the sample tubes from collection through Earth return. Therefore, immediately after landing and regardless of the temperature selected, the spacecraft should be recovered and placed in a container designed to control and stabilize its physical condition and surrounding environment and monitor its internal temperature conditions; this container should be designed to also provide biosafety in case of a nonnominal landing. Once the optimum temperature for the RSTA has been defined, the process should be designed to maintain the sample at this temperature and reduce fluctuations during shipment while expediting transport to its destination, the SRF. For instance, from analogy with certain terrestrial mineral and biomineral phases, reversible and irreversible phase transitions may occur under different $\mathrm{T}$ and/or mineral phases that could be destroyed and/or new minerals formed (Tosca et al., 2021). It would be important that both the $\mathrm{T}_{\text {optimum }}$ of the RSTA and the speed of the cooling process are carefully controlled to avoid, for example, mechanical damage (Libourel et al., 2021). Although, in principle the external temperature fluctuations should not compromise the integrity of the sample stored in the tubes, and thus we should consider that some tubes may not have intact seals. Therefore, the entire RSTA, even compromised tubes, should be maintained at the optimum temperature during transport from the landing site to the SRF to prevent any further damage to the samples with failed seals.

Moreover, there are many variables that could influence the stabilization of $\mathrm{T}_{\text {optimum }}$ as well during the logistics process and, finally, impact costs that could include the following:

- seasons and transit time: for example, summer versus winter variations coupled with long transits can make it difficult to stabilize the $\mathrm{T}$, particularly if the $\mathrm{T}_{\text {optimum }}$ is extremely cold. To minimize the risks due to $\mathrm{T}$ fluctuations and associated costs, the logistic process should be planned to minimize transit times.

- tubes: it is necessary to evaluate whether the tubes (and thus the samples within) will be super-heated or will remain at their estimated Mars collection temperature during Earth atmosphere entry and descent.

Increased reaction rates do occur with higher temperature; however, the samples may have been heated to temperatures approaching or exceeding room temperature prior to delivery during sample acquisition (drilling), while cached on the 
martian surface, during Earth entry, etc. Thus, if a lower temperature were to be chosen, there would be no a priori way to choose what that temperature should be. The MSPG2 Curation group suggests that the $\mathrm{T}_{\text {optimum }}$ should be gradually stabilized during transport from the landing site to the $\mathrm{SRF}$, and that the $\mathrm{T}_{\text {optimum }}$ in transit should be $15-25^{\circ} \mathrm{C}$, the proposed room temperature of SRF sample handling.

FINDING C-2: Immediately after Earth landing, the spacecraft would be recovered and placed in a container designed to control and stabilize its physical conditions. The optimum temperature ( $\left.\mathrm{T}_{\text {optimum }}\right)$ of the sample tubes during transport to the Sample Receiving Facility (SRF) should be the same as the operating temperature of the SRF to avoid unnecessary temperature shock.

2.1.2. Temperature of the samples within the SRF. Temperature and the relative humidity of the samples need to be monitored and measured while in the SRF. To store all the Mars Sample Return samples at colder temperatures $\left(-20^{\circ} \mathrm{C}\right.$ or lower) introduces significant challenges and costs to the facility and sample handling/processing procedures. Depending on what samples are collected and cached by Perseverance rover, a future group may determine a compelling reason to store a sample tube or small subset of sample tubes in cold storage, but the SRF should not be designed for cold sample processing, particularly in a BSL-4 setting. Therefore, we recommend moving most or all the samples to room temperature $\left(\sim 15-25^{\circ} \mathrm{C}\right)$ as soon as they are received at the SRF and holding them at that temperature. The option for cold storage of subsamples should be available in the SRF should the need arise, which is outlined in a companion document related to time sensitive science (Tosca et al., 2021).

We have libraries full of data on rocks from around the solar system that have been collected and stored at room temperature. Currently, almost all curated extraterrestrial samples are stored at room temperature (Russell et al., 2019), and all instrumentation planned for BC and PE are designed to operate at room temperature. We know exactly how our scientific instruments operate at that temperature. If samples were stored at cold temperatures, in most cases the operators would need to bring the samples to room temperature prior to making the observations or analytical measurements. Temperature fluctuations between cold storage and room temperature for transport and initial sample characterization measurements could be more damaging than staying at a consistent room temperature. One example would be the possibility of atmospheric vapor condensing on the samples during the analytical measurements.

Alternatively, BC and PE would need to be done at cold temperatures. To minimize the chance of temperature variations, all the samples, instruments, and the operator would need to stay in $-20^{\circ} \mathrm{C}$ conditions, which would mean the operators would not only have to consider Biosafety Level-4 PPE requirements, but also contend with cold temperatures, the complexity of sample handling at these temperatures, and potentially hypothermia. This would also dramatically increase the complexity of the measurements, which would likely slow down the sample catalog preparation and, in turn, acquisition of scientific results from the samples.
Therefore, we should not plan for BC and PE measurements at cold temperatures. We did not talk about triboelectric charging or humidity and their relation to sample handling, and thus we suggest that a future group considers this (see Section 8.1, Future Work 1).

FINDING C-3: The Sample Receiving Facility (SRF) should operate at room temperature $\left(\sim 15-25^{\circ} \mathrm{C}\right)$ and the samples should be held at this temperature through all steps of initial sample characterization, with the option for cold storage of subsamples available in the SRF when needed.

\subsection{Sample tubes}

On Mars Perseverance there are 43 sample tubes, each roughly 14 centimeters long and 2 centimeters across (Moeller et al., 2021) (Figure 1). M2020 will carry enough supplies to fill and seal 38 sample tubes, the remaining five are pre-configured blanks (Moeller et al., 2021). The Orbiting Sample container (OS) can hold 30 tubes (based on current designs) and that includes both natural samples and blanks. Some tubes will serve as witness tubes, filled with material such as aluminium mesh or ceramics to trap environmental contaminants. The sample tubes have an interior titanium nitride coating, which is a specialized surface treatment that resists contamination (Moeller et al., 2021). Payload Cavity, also known as the bore, is the area in the tube where cores of martian rock and samples of regolith would be stored. The plunger works in concert with the spring to release (retract) or activate (extend) the two exterior-mounted ball locks. Springs, along with the plunger, act to release or activate the ball locks. The Hermetic Seal is a mechanically activated plug designed to ensure that no contaminants can get into the sample tube, and they are engineered to a specified leak rate. As specified in the key assumptions, the term "sample tube" is synonymous in this document to the term Returnable Sample Tube Assembly (RSTA) commonly used by the M2020 engineering team.

\subsection{Hardware de-integration}

The OS, which is designed to carry the sample tubes, would be captured by a Sample Return Orbiter (SRO).

"A Rendezvous and OS Capture System (ROCS) is proposed as a payload for an SRO spacecraft to perform OS capture in Mars orbit, transfer of the OS to an EEV and release of an EEV towards Earth for Earth entry. The ROCS would seal off all unsterilized Mars material within both Primary and Secondary (redundant) Containment Vessels (PCV and SCV) to meet potential to-be-defined Planetary Protection Restricted Category V sample return mission containment assurance policies (Figure 2). The Containment Vessels separate the region exposed to Mars material from the "Earth clean" region isolated from Mars material and a coil assembly that performs the brazing. The ROCS system consists of an Aeroshell installed with the SCV Base, an SCV Lid, and an Aeroshell Lid. Connecting the SCV Lid to the PCV Base, as well as the Aeroshell Lid to the Aeroshell occurs along the EEV reference axis" (Figure 3; Younse et al., 2018). 


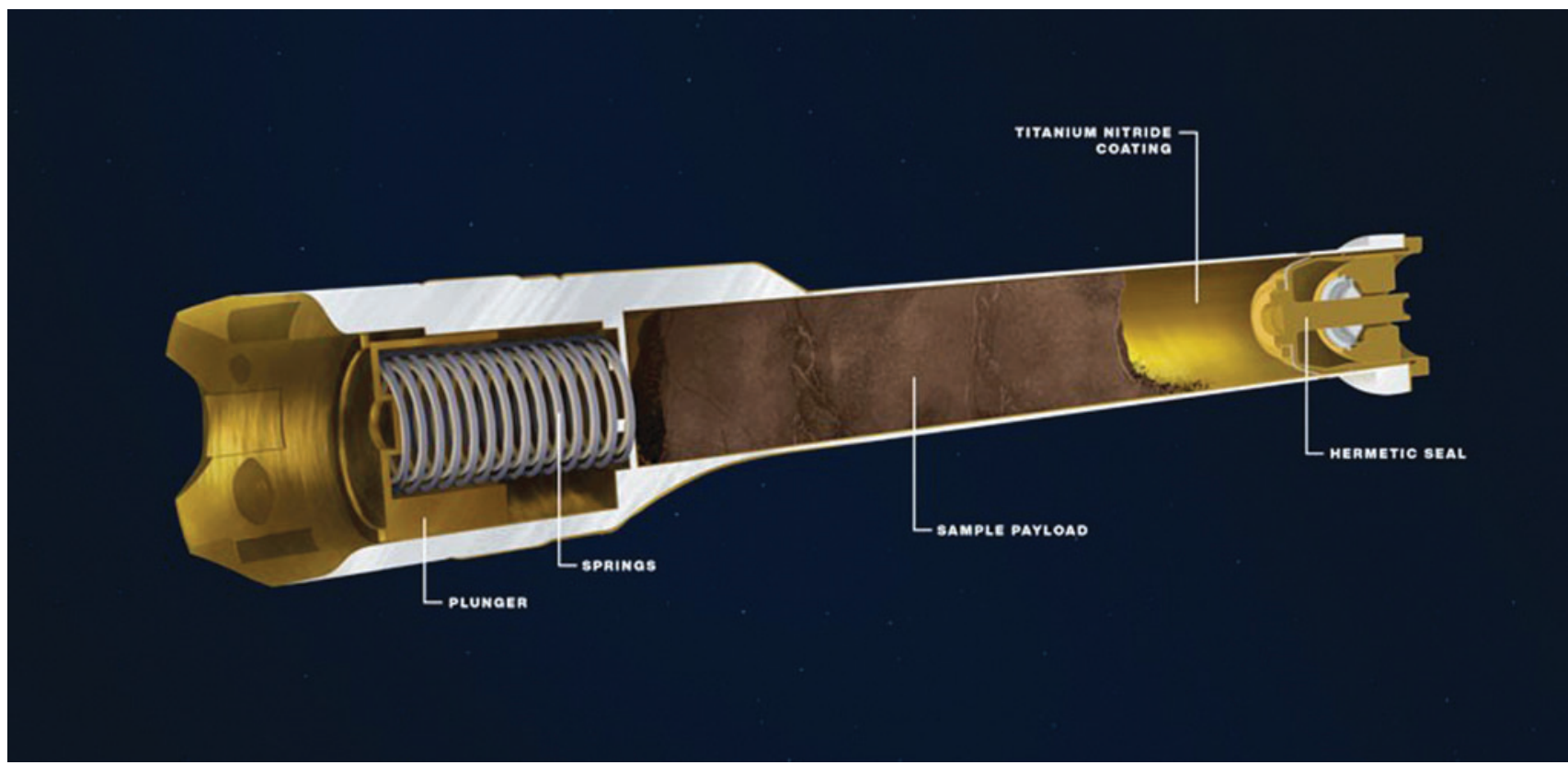

FIG. 1. This illustration depicts the interior of a sample tube being carried aboard the Mars 2020 Perseverance rover. About the size and shape of a standard lab test tube, the 43 sample tubes headed to Mars are lightweight, hardy enough to survive the demands of the round trip, and so clean that future scientists will be confident that what they are analyzing is $100 \%$ Mars, without terrestrial contaminants. Credit: NASA/JPL-Caltech.

Following a year-long return trip to Earth, ERO would deploy the EEV for touchdown at a landing site to be determined. Once the EEV has been recovered from the landing site, it would be transported to the Sample Receiving Facility. The EEV would then be progressively opened to get to the a

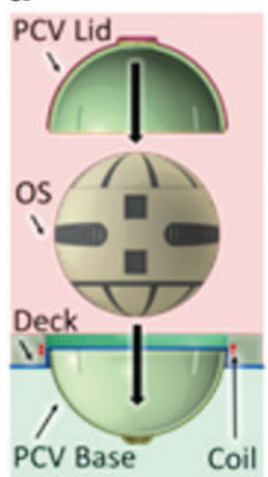

b

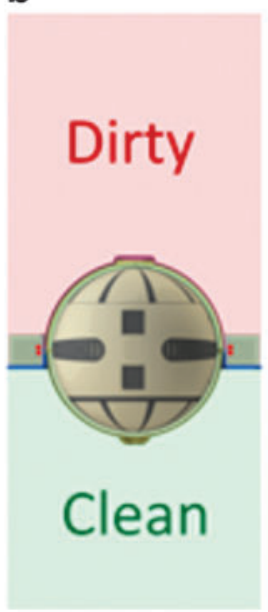

C

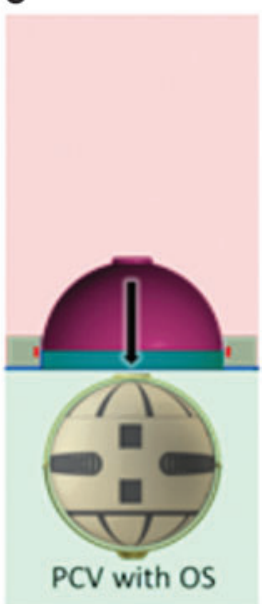

FIG. 2. Illustration of an inductive brazing sequence. (a) OS assembled with the PCV Lid to the PCV Base brazed to the Deck. (b) Inductive brazing operation simultaneously brazes together the PCV Lid outer wall to the Deck, brazes together the PCV Lid inner wall to the PCV Base, separates the PCV Lid inner wall from the PCV outer wall, separates the PCV Base from the Deck, and sterilizes all regions along and in between the brazing surfaces. (c) PCV Lid inner wall, the OS, and the PCV Base removed from the Deck (Younse et al., 2018). sample tubes. The outer parts of the spacecraft, which are part of the ERO - the Earth Return Orbiter, should be dust-free and only interesting from a contamination knowledge standpoint having never been to the martian surface.

Removing the outer sample vessel from the EEV would need to be done in a controlled laboratory environment, especially once the SCV starts to be removed. This would also allow for analysis of the gas between the SCV and PCV, to verify that the seal held. It is particularly important to open the PCV in a controlled environment of an isolator because this is where the Mars dust samples would begin to be collected, and it is important to avoid the terrestrial environment during this time. The OS would have been loaded with samples at the martian surface (as part of SRL-the Sample Retrieval Lander), so its interior and exterior surfaces are likely to have dust on them (Figure 4). The sample tubes almost certainly would have a coating of dust, since they would have been lying on the martian surface for a period, unless cleaned on Mars. A pristine OS isolator would be needed to open the OS, collect the dust on the outside of the OS, and remove the sample tubes (see Section
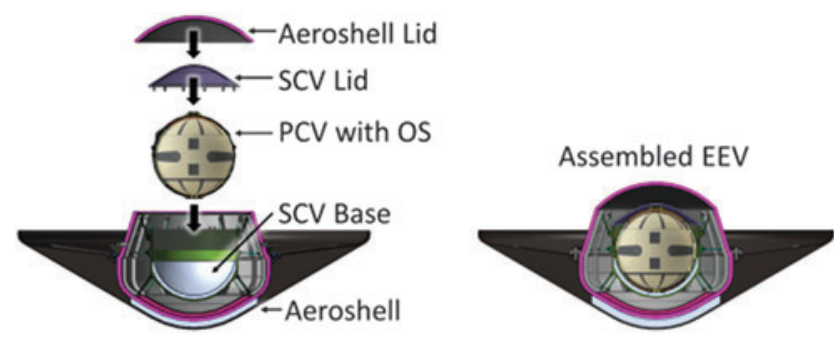

FIG. 3. The Earth Entry Vehicle assembly (Younse et al., 2018). 


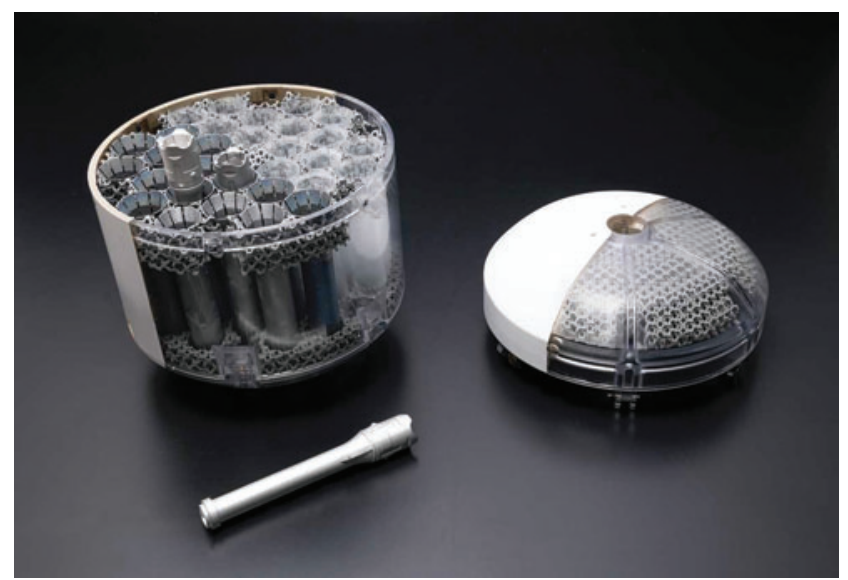

FIG. 4. This image shows a concept model of NASA's orbiting sample container, which would hold tubes of martian rock and soil samples that would be returned to Earth through a Mars sample return campaign. At right is the lid and a model of the sample-holding tube is shown at bottom left. The sample container would help keep contents at less than about $30^{\circ} \mathrm{C}$ to help preserve the Mars material in its most natural state. Photo Credit: NASA/JPL-Caltech.

2.4.1). This isolator would be the long-term home for the OS after the sample tubes are removed. This pristine isolator would need to have windows and/or ports for visual inspection of the hardware, and there should be glove ports for doing the disassembly (unless it is decided that the disassembly should be done robotically).

\subsection{Isolator details}

There would need to be a series of "pristine" and "nonpristine" isolators available for the hardware de-integration; sample preparation; SSAP; and Pre-BC, BC, and PE steps on the MSR samples. It is important to distinguish the differences between these types of isolators and know their respective requirements.

2.4.1. Pristine isolators. When the samples tubes are open, they should be in an extremely clean, aseptic (the level to which will be defined later) and well-regulated condition that is consistent throughout all the pristine isolators in the sample receiving facility. There should be a limited and controlled list of materials requirements for components and tools within the pristine isolators. Instruments should not be allowed in the isolators because of contamination risks (unless modified to meet the materials restriction list), though analyses could be performed through ports and windows on the isolators. Samples or (much more commonly) portions of samples that leave pristine environments should NOT be allowed back into pristine isolators or containers.

KEY DEFINITION: Pristine Sample Containers: Clean controlled environments (isolator/glovebox/isolation chamber) with a controlled atmosphere where BC would take place. A limited controlled list of materials would be allowed. Once samples are removed from a Pristine environment, they should not be allowed back into Pristine sample containers.
2.4.2. Non-pristine sample containers. Non-pristine containers (isolators/gloveboxes/storage containers, etc.) would be for long-term storage of samples and subsamples that have left pristine environments or were never exposed to a pristine environment. These areas are largely for clean storage, and any sample processing of these materials would likely be done in either the macrosample or microparticle processing labs. Non-pristine environments have a controlled (but not highly restrictive) list of materials requirements for components and materials allowed within them. These are clean environments (the exact level of cleanliness will be defined later) with or without a controlled atmosphere (this is TBD and may depend on unique needs of MSR). Once a subsample leaves its pristine container, it is barred from re-entering its pristine container, in that it may contaminate the portions of the same sample that have remained in the pristine environment. Consequently, there would be a need for non-pristine sample containers to house samples that have left their pristine environment but were not consumed during analysis (either by sample safety assessment, scientific analysis in containment, or PE). Hence samples or portions of samples that leave nonpristine containers are allowed back into non-pristine containers. At least two non-pristine isolators would be required for curation activities. This number may change as the Perseverance rover starts to drill samples of different types. It should be noted here that more isolators would be required to conduct SSAP activities and other time-critical science.

KEY DEFINITION: Non-Pristine Sample Containers: Clean environment (isolator/glovebox) with, or without, a controlled atmosphere for samples removed from Pristine Containers and for those samples that were never in Pristine Containers. Limited (but not highly restrictive) list of materials would be allowed. Once samples are removed, they would be allowed back into their Non-Pristine Containers.

2.4.3. Cleaning, sterilizing, and monitoring. Cleaning and sterilizing curation sample handling tools, containers, and other equipment (such as gloveboxes, isolation chambers, and desiccators) is required for the curation of astromaterials. The facility shall include the capability of doing precision cleaning to meet the Contamination Control (CC) needs of the SRF. Precision cleaning is typically required where equipment is cleaned to a specified cleanliness and the cleanliness is measured and verified to a standard. During final cleaning, specialized equipment is needed to purify the aqueous cleaning solutions. Ultrapure water (UPW) is typically the final cleaning agent and requires substantial initial investment to purchase a 3,785 liter water tank and monthly maintenance cost. In addition to the pre-clean and final clean labs, there would also need to be a set of scientific instruments that can be used to verify and validate $\mathrm{CC}$ requirements in the facility. With this there will need to be a way to verify sterility. For example, we would need to regularly check for particle counts within clean lab spaces, monitor the trace gases in the curation-grade gases used in the processing cabinets, as well as check the isotopic compositions of these gases and of the UPW. We will need to monitor for microbial contamination in the UPW, air filter systems, labs, and within the gloveboxes. We will also need to 
set out witness plates to monitor for microbial, organic, and inorganic contamination in spaces where pristine samples are handled. Having the ability to do this in house will provide efficiency to cleaning procedures and provide flexibility to dynamic schedule needs and time pressures. It will also minimize the time between noticing a problem and being able to react to it. A more detailed $\mathrm{CC}$ plan will need to be developed in future working groups (see Section 8.1, Future Work 2).

2.4.4. Gas to interact with the samples. It would be crucial to minimize the number of gases that come into direct contact with samples, and these gases need to be high purity and consistent throughout the pristine isolators. Samples at the Johnson Space Center, for example, are stored under high purity gaseous nitrogen $\left(\mathrm{N}_{2}\right.$ gas), and there is a gas production facility on site to minimize the need for constant delivery (and potential delays) (McCubbin et al., 2019). Dry $\mathrm{N}_{2}$ gas has not been a problem for $\mathrm{N}$ isotope studies for high- $\mathrm{T}$ release phases, so that should be acceptable as a standard, but we may consider an additional inert atmosphere such as argon (Ar) for samples where we are particularly concerned about low- $\mathrm{T}$ release of $\mathrm{N}$ from bulk sample analysis. This is not likely to be an issue for organic studies because they are either in situ or go through a step of separating out soluble and insoluble organics where atmospheric contamination is not a significant concern. The case in which Ar would be needed is rare but should be considered. We recommend that a future working group study the pros and cons of different curation-grade gases that could be used within all the isolators (See Section 8.1, Future Work 3).

2.4.5. Approved materials for contact with the Mars returned samples. It would be crucial to minimize the number of materials that come into direct contact with samples to minimize contamination yet still be able to process the samples relatively easily. Dedicated manufacture guidelines and material of instruments and tools to be used shall be defined to limit the risk of contamination by such instruments. Performing BC and PE steps is critical for producing the sample catalog, but experiments proposed by the PIs that could be compromised by inorganic, organic, and/or biological contamination need to be evaluated. The NASA Johnson Space Center (JSC), Astromaterials Acquisition and Curation Office has an approved list of materials for curating sample return missions (Apollo samples, Hayabusa, etc.) and samples recovered from Antarctica. These are 304L/316L stainless steel, Teflon, and T6061 aluminium (McCubbin et al., 2019). The Curation Office also uses Neoprene and Hypalon or chlorosulfonated polyethylene for gloveboxes, but this glove material never comes in direct contact with curated samples. These three materials optimize the benefits from the perspective of organic and inorganic contamination, while also being highly functional for sample processing. For example, Day et al. (2018) studied the stainless-steel tools that are used at NASA JSC and determined that $>0.01 \%$ by mass contamination is needed to affect Highly Siderophile Elements (HSE) in Apollo samples, and there is no indication that contamination from stainless steel tools has affected HSE data from Apollo samples, so this likely is a suitable material for the MSR samples (Day et al., 2018). All these materials could also be sterilized against biological contamination. It is important that materials type be optimized for all needs across all sample types to reduce the number of required pristine isolators. The sample tubes are made of $\mathrm{Ti}$ (Moeller et al., 2021), so Ti may be an acceptable material for making tools, but the minor and trace element abundances of 304 and 316 stainless steel are well known and do not inhibit scientific investigations of metals, including HSE. More work is needed to determine whether the same is true for Ti alloys. We recommend that a future working group work on optimizing the list of materials that would be suitable for the MSR samples (see Section 8.1, Future Work 3).

\subsection{Overview of analytical instruments for curation}

Documentation of the Mars returned samples in the SRF should consist of three sequential stages: Pre-BC, BC, and $\mathrm{PE}$, which together can be called "initial sample characterization." All samples would go through Pre-BC and BC, but not all samples would necessarily go through PE. The reason for this is that, in $\mathrm{PE}$, some instruments could be damaging to some investigations. The material used in PE would be documented as such and not used for allocation to non-destructive types of investigations; material not subjected to PE damage would be reserved for allocation in support of non-destructive investigations.

A significant portion of the Curation FG's efforts was determining which instrumentation would be required to produce a sample catalog for the scientific community and how and when it should be used (Figure 5). The goal is to provide enough information for the PIs to request material for their studies but stopping short of doing targeted scientific research that would be left to peer-reviewed competitive processes. We reviewed the proposed scientific objectives of the International MSR Objectives and Samples Team (iMOST) (Beaty et al., 2019) to make sure that the instrumentation suggested would be sufficient to cover these key science planning questions (Table 1; Section S-6). It was determined that, for Pre-Basic Characterization, two instruments are required, a Magnetometer (see Section S-1.1) and X-ray Computed Tomography scanner (XCT see Section S-1.2). For Basic Characterization, there are four instruments that were necessary, which are 1) an analytical balance (see Section S-2.1), 2) a binocular microscope (see Section S-2.2), 3) multispectral imaging, and 4) hyperspectral scanning (see Section S-2.3). Then in Preliminary Examination, there are proposed to be eight instruments that are available, when necessary, for more detailed information for the sample catalog. These are 1) Variable Pressure-Field Emission Scanning Electron Microscope (VP-FE-SEM, see Section S-3.1), 2) Confocal Raman spectrometer (see Section S-3.2), 3) Deep UV Fluorescence (see Section S-3.3), 4) Fourier Transform Infrared Spectrometer (see Section S-3.4), 5) Micro X-ray Diffractometer (see Section S-3.5), 6) X-ray Fluorescence Spectrometer (see Section S-3.6), and 7) Petrographic Microscope (see Section S-3.7). An overview of how these instruments align with the goals of sample early characterization is summarized in Table 1.

\section{Pre-Basic Characterization (Pre-BC) Activities}

These activities constitute the removal of the dust and checking the sample seals TBD (see also Tosca et al., 2021) as well as measurements that would be performed on sample 


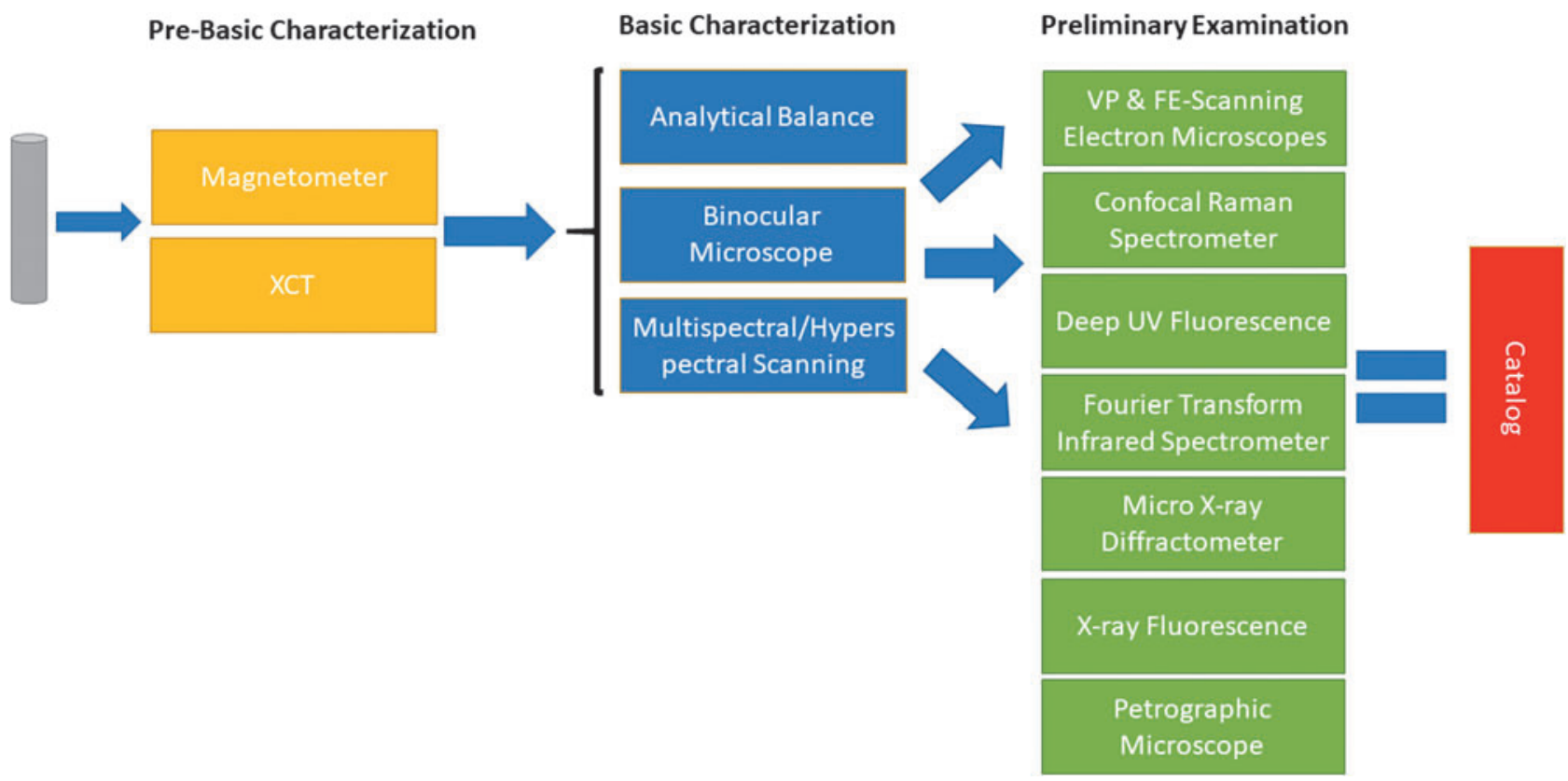

FIG. 5. Proposed list of instrumentation for Pre-Basic Characterization, Basic Characterization, and Preliminary Examination to produce a sample catalog.

tubes before they are opened and before they would be placed within a pristine isolator (which marks the start of BC). Pre-BC measurements should occur on all the samples, unless it is decided to not open some sample tubes. These measurements should consist of minimally destructive techniques, particularly if they are applied to the entire sample. The motivation behind Pre-BC measurements is to 1) conduct measurements that would be lost or compromised during the opening of the tubes and 2) conduct measurements that would inform how the tubes are opened, processed, and subsampled during BC. Several Pre-BC measurements have already been identified as critical, including dust removal, oriented bulk magnetic measurements, and XCT (Figure 5). Other analyses could be added to this list as requirements become clearer. The primary objectives of Pre-BC would be the following:

- Carefully describing and removing the dust from the exterior of the sample tubes;

- Checking the sample tube seals;

- Collection of the primary, or baseline magnetic measurements of the samples;
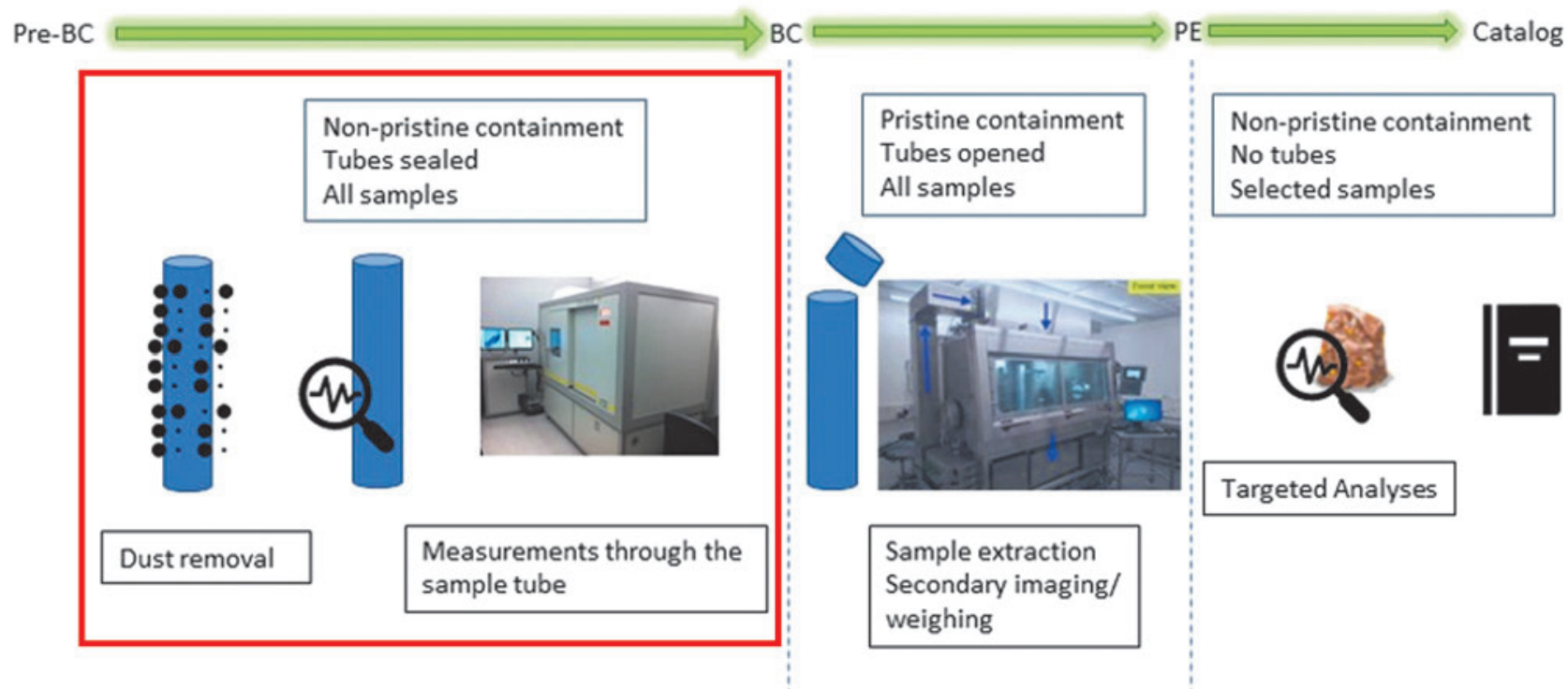

FIG. 6. Proposed sequence of activities within the Pre-Basic Characterization (Pre-BC), Basic Characterization (BC), and Preliminary Examination (PE). 
- Penetrative imaging of the samples in the tube, to add information to the sample dossier (information from the M2020 Rover) and help inform the sequence of tube opening.

MAJOR FINDING C-4: Measurements on all the sample tubes before they are opened are essential to conduct as the samples could be compromised upon opening of the tubes. This step is called Pre-Basic Characterization (Pre-BC). These are measurements that would inform how the tubes are opened, processed, and subsampled during Basic Characterization (BC).

\subsection{Envisioned processes and workflows}

Once the sample tubes are removed from the OS, Pre-BC starts. The first step in Pre-BC would be removing the dust from the exterior of the sample tubes (Section 3.2.1.). Before the dust is disturbed, detailed observations of the material should be made, including macroscale observations of the dust and imaging. After the dust is carefully removed and stored in a non-pristine isolator, the sample seals should be checked, and the headspace gas secured (see Section 4.3. and Tosca et al., 2021). Once the sample seals are checked, the sample tubes should be placed into a secondary sample holder (see discussion on Sample Tube Isolation Chambers, STICs, Section 3.2.3.) to prevent (or minimize) interaction of the gas within the sample tube and the environment. After the samples are placed in their secondary sample holder, magnetic measurements and XCT measurements would be carried out. The XCT measurements would also inform SSAP, and this is a time where Curation and SSAP need to interact and prioritize the order in which the sample tubes are processed. After the imaging and magnetic measurements are complete, the secondary containers should be thoroughly cleaned and prepared to enter the pristine isolator, which marks the start of BC. Without a wellconstructed and curated Contamination Knowledge (CK) collection, the baseline for contamination within the returned samples cannot be established (Harrington et al., 2018), so we would like to call out its importance within this document.

\subsection{Workspace configuration}

There would need to be at least two non-pristine isolators available for processing the dust on the outer portions of the sample tubes concurrently. The start of the Pre-BC process could be a bottle neck, as there will be a need to start the process of getting sample tubes through this stage quickly. We have identified the need for at least one XCT for this step but could consider more than one to minimize any delays that could arise due to instrument downtime and to increase throughput of samples tubes through this stage, as there will be concurrent SSAP and Curation needs for this instrument. There will need to be workspaces for computers to operate the XCT, as well as high end data storage available for processing and manipulation of the data from the XCT.

3.2.1. Sample tube dust removal. There is a science rationale for the removal of the dust on the outside of each sample tube once it is removed from the OS but before it is placed into the STIC (Grady et al., 2021). The isolator would need to have the ability to remove tiny dust grains from the sample tubes. There are several methods by which this could be done, and there are some important lessons learned from JAXA's first Hayabusa mission and NASA's Genesis mission (e.g., Calaway et al., 2009). We suggest developing a quick way of removing the dust that does not negatively impact the samples within the tubes and that minimally changes the dust sample on the outside of the tubes. For example, we suggest avoiding the use of a liquid to remove the dust, if possible, even though that may be one of the quickest processes to remove dust. JAXA has developed numerous techniques to handle and remove dust from flight hardware, and their lessons learned could be incredibly useful to MSR for developing dust removal strategies for the dust on the outsides of the tubes. Two processes of dust removal that have been discussed include swabbing the outsides of the tubes with Teflon or tube vibration to remove the particles. The efficacy and effects on the samples within the tubes need to be evaluated before either process would be deemed acceptable. The dust would have been exposed to a contamination environment that is less controlled than the samples in the tube interiors, so the dust samples should be stored within nonpristine containers. More work is needed to find the best dust removal option, and we suggest that a future working group focus on this (see Section 8.1, Future Work 5).

MAJOR FINDING C-5: Careful collection and storage of the serendipitous dust on the outside of the sample tubes is a critical step in the curation process in the Sample Receiving Facility. The dust collected is a valuable resource to the scientific community.

3.2.2. Sample seal integrity. We would like to be able to measure the leak rates on the sample tubes as quickly as possible after the OS is opened. For reference, the engineering test program showed that the leak rate is likely to be less than 1 x 10-8 scc/s, though it cannot be guaranteed (Swindle et al., 2021). This leak rate has been calculated such that the amount of leakage that occurs during a two-year period exposed to the vacuum of space is equivalent to about $5 \%$ of a sample gas - this is deemed to be marginally acceptable for the sample to still be useful scientifically. However, an engineering seal test program showed that actual seal/leak values can range by about three orders of magnitude (in both directions) about this value. The best samples will be the ones for which the seals on the sample tubes have the lowest leak rate, and that needs to be a documented part of the sample catalog. Unfortunately, we have not been able to identify a way to make this measurement without getting access to both sides of the seal (for example, this is how a He leak detector works). It may not be possible to make this measurement effectively while the natural sample gas is still inside the tube. It may be necessary to extract the gas, then evaluate the quality of the seal based on the composition (diagnostic could be, e.g., the Ar isotopes or the $\mathrm{CO}_{2} / \mathrm{O}_{2} / \mathrm{N}_{2}$ ratios). Perhaps a "post-mortem" test (after the samples are removed) of the sample seals will be the best way to determine the leak rate. In any case, this is a topic best left for a future study team (see Section 8.1, Future Work 6). 
MAJOR FINDING C-6: Careful collection and storage of the unaltered and unfractionated headspace gas collected from the sample tubes is a critical step in the curation process in the Sample Receiving Facility. The gas collected is a valuable resource to the scientific community.

3.2.3. Individual sample tube isolation containers. The sample tubes were not designed for long-term storage, and integrity of the seals could be compromised. However, the interaction between Earth atmospheric gases and the gases that are in the sealed tube should be minimized (see Swindle et al., 2021 for more details). We propose a conceptual device that could house the sample tubes once the dust has been removed from the exterior of the sample tubes, called the Sample Tube Isolation Container (STIC).

The STIC serves as a secondary vessel that minimizes gas exchange between Earth's environment and the gas inside the sample tube. The STIC also serves to isolate each of the tubes from each other so that multiple tubes could be stored within the same pristine isolator. The types of samples that can be stored together are discussed later in the BC portion of the document (Section 4). The STIC should be at least as clean as the pristine isolators. It is foreseen that the tubes or subsamples may need to leave biocontainment for transport and/or analysis. Whether biocontainment in transport should be provided by the STIC, or by an additional container, should be the subject of future consideration.

The STICs could be designed in such a way that the headspace gases in the tubes can be extracted while they are contained within the STIC. One concept we discussed was that each sample tube container have a gas sampling/extraction mechanism that can be connected to a gas manifold system capable of extracting and measuring the pressure and composition of the gas. By having each tube within its own dedicated isolation chamber designed for long-term storage, we would be able to select which samples, if any, would be saved for the future. For example, some Apollo samples have never been opened, and a subset of those are being opened 50 years later as part of the Apollo Next Generation Sample Analysis Program (ANGSA). As the number and types of samples are confirmed from the Mars2020 team, more discussion should occur on whether all the samples would go through Pre-BC and BC in the SRF, or some would remain sealed. This will largely be driven by Planetary Protection requirements for release of the samples from the SRF. More thorough design and thought must be given to this concept, and we suggest a future working group be assembled to do this (see Section 8.1, Future Work 8). Once the sample tube has been opened, the samples may or may not be placed back into the STIC for long-term storage.

FINDING C-7: To minimize the interaction of Earth atmospheric gases and gases that are in the sealed sample tubes, once the dust is removed from the exterior of the sample tubes, they should be placed into individual sample tube isolation chambers (STIC) as quickly as possible.

\subsection{Pre-Basic Characterization Summary}

There are two critical measurements that have been identified as required for Pre-BC; an X-ray Computed Tomography and a magnetometer. The challenge in Pre-BC is that the samples would be inside sealed titanium tubes; we recognize only two instruments that can make meaningful measurements through the tube walls without causing unacceptable damage (note that higher-energy forms of penetrative imaging also exist and are discussed in a supplemental Section S-5). Both instruments are required for this stage of initial sample characterization, and the information gained feeds into the BC and PE stages of sample characterization. In sum, the instrument options for doing through tube characterization during the Pre-BC phase is highly limited, and the two instruments named above are considered absolutely essential.

FINDING C-8: There are compelling reasons to perform penetrative $3 \mathrm{D}$ imaging prior to opening the sample tubes. A laboratory-based X-ray Computed Tomography scanner is the best technique to use and the least damaging to organics of the penetrative imaging options considered.

\section{Basic Characterization (BC)}

Basic characterization (BC) is a series of data collection steps that do not (or minimize to the greatest degree possible) alter, damage, or induce any change in the sample and its associated properties (physical, chemical, spectral etc.). These steps would include weighing, photographing, and optical investigation (Figure 7). The BC steps would be applied to every sample in a standard way and would be used to inform the PE and SSAP methods that would be implemented. There is information from M2020 that will be available before opening the tubes, as rover data and geological setting information should be collated beforehand, including (but not limited to) sample type and likelihood of the presence of organic molecules. This information would be integrated into the $\mathrm{BC}$ process and referred to when designing the PE protocols for each sample. It is during this stage that the sample tubes would be opened within the pristine isolators for the first time, which starts the clock for time-sensitive measurements (in particular, the mineralvolatile exchange processes); detailed observations should be made for these samples (see Tosca et al., 2021 for more details).

MAJOR FINDING C-9: Measurements on all the samples once the sample tubes are opened within the pristine isolators are essential to make initial macroscopic observations such as weighing, photographing, and optical observations. The first step to this stage is removal and collection of the headspace gas, which then starts the clock for time-sensitive measurements. This step is called Basic Characterization (BC). 
Pre-BC

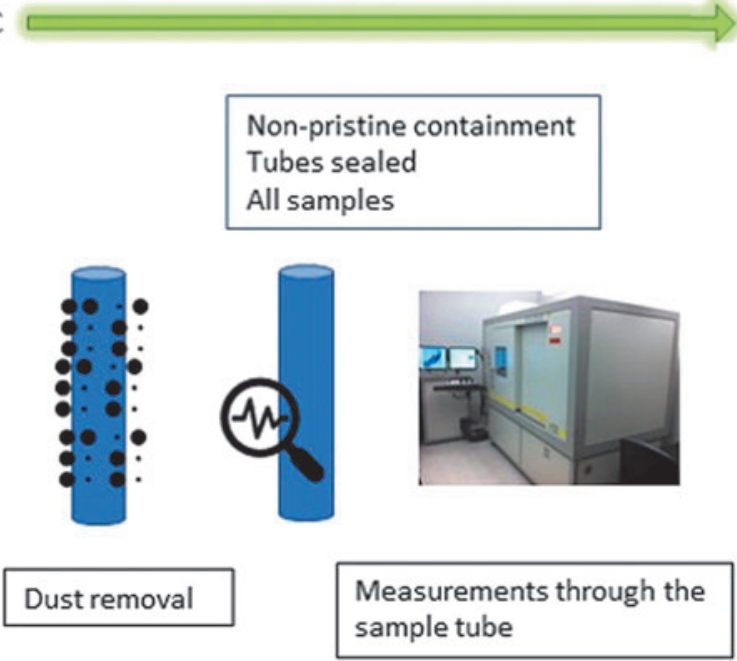

$B C$

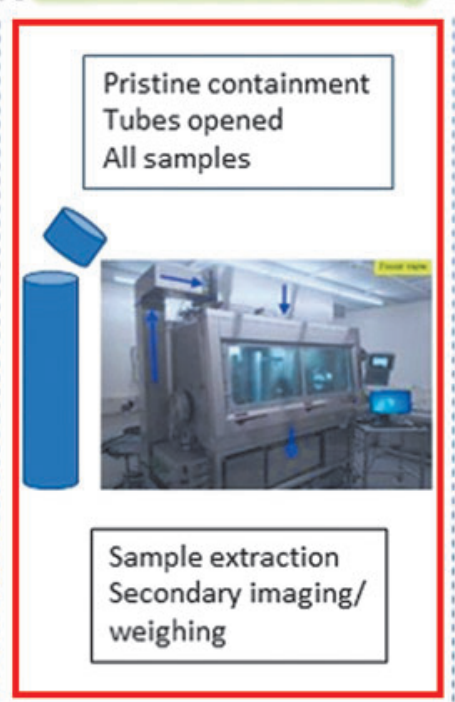

Catalog

Non-pristine containment

No tubes

Selected samples

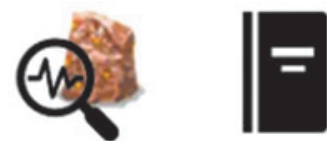

Targeted Analyses

FIG. 7. Proposed sequence of activities within the Pre-Basic Characterization, Basic Characterization, and Preliminary Examination, with the Basic Characterization stage enclosed within a red box.

\subsection{Envisioned processes and workflows of Basic Characterization}

Once in the pristine isolator, the sample tubes will be opened and exposed to the curation gas environment. The Johnson Space Center uses nitrogen $\left(\mathrm{N}_{2}\right)$, as it is relatively inert and does not oxidize or alter the samples. It is compositionally simple (i.e., not a gas mixture or molecule with more than one element like $\mathrm{CO}_{2}$ ), so it minimizes the number of elements that could be compromised by its use in the curation environment. It is a consumable, so it becomes a major cost to maintaining the facility. Liquid $\mathrm{N}_{2}$ is produced at a high purity for other purposes at an industrial scale, which keeps costs of high quality $\mathrm{N}_{2}$ low compared to other options like Ar. But the gas used to backfill the sample tubes and what is used in the isolators can be determined later (see Section 8.1). Any time-sensitive measurements could occur at this point, and/or (sub) samples will be identified for alternative storage, such as flash freezing for future analytical measurements or possible residual gas monitoring after headspace gas extraction (see Tosca et al., 2021 for this discussion).

\subsection{Number of isolators and workspace required}

We will want to minimize cross contamination between the sample tubes. It is anticipated that Jezero Crater will have, at minimum, carbonate-rich rocks, non-carbonate mudstones and sandstones, igneous rocks, impact breccias, and loose dust and regolith. One way to isolate the samples would be a set of isolators for each rock type where cross contamination would compromise scientific investigations. Additionally, the blanks and witnesses should have their own set of isolators (one for the drillable blank, one for the witness tubes, and one backup that can be used for either witness tubes or blanks). Based on the rock types we anticipate being collected in Jezero Crater, we should have, as a preliminary conclusion, an isolator set for carbonate-rich rocks, a set for non-carbonate-rich sedimentary rocks, a set for igneous rocks (including impact breccias, impact melt rocks and/or silica-rich rocks), and a set for loose dust and/ or regolith which will be updated as M2020 collects the samples. Each isolator should be cleaned before and after a sample is opened and/or processed to prevent cross contamination between samples in general. Consequently, it will be advantageous to have at least two isolators for each sample type to minimize down time between sample changes. Although our basis for grouping samples into isolator types is on rock type, there are other factors that could be used to make such groupings like atmosphere or materials requirements. Another way would be to split the samples up by bit, as there are no cleaning plans on Mars between the drill bits, so there will be cross contamination on Mars between those sets of samples. A full description of drill bits can be found in the work of Moeller et al., 2021. As the samples are drilled, it will become clearer on how best to group the samples. Even if there are no cross-contamination concerns between samples, and all sample isolators are comprised of the same materials and atmosphere, schedule and workflow considerations will also affect the number of different sets of isolators that could be worked on in parallel. Finally, it will likely be an arduous process to clean and sterilize isolators. Although a procedure has not been determined, cleaning could take weeks or months based on existing procedures for clean room glove boxes that are not BSL-4 rated in current curation clean labs.

FINDING C-10: To avoid cross contamination between samples, it is recommended that, for processing through the isolators, the samples be organized into groups that have like properties. Given what we know about the geology of Jezero Crater, a reasonable starting assumption is five such groups. 


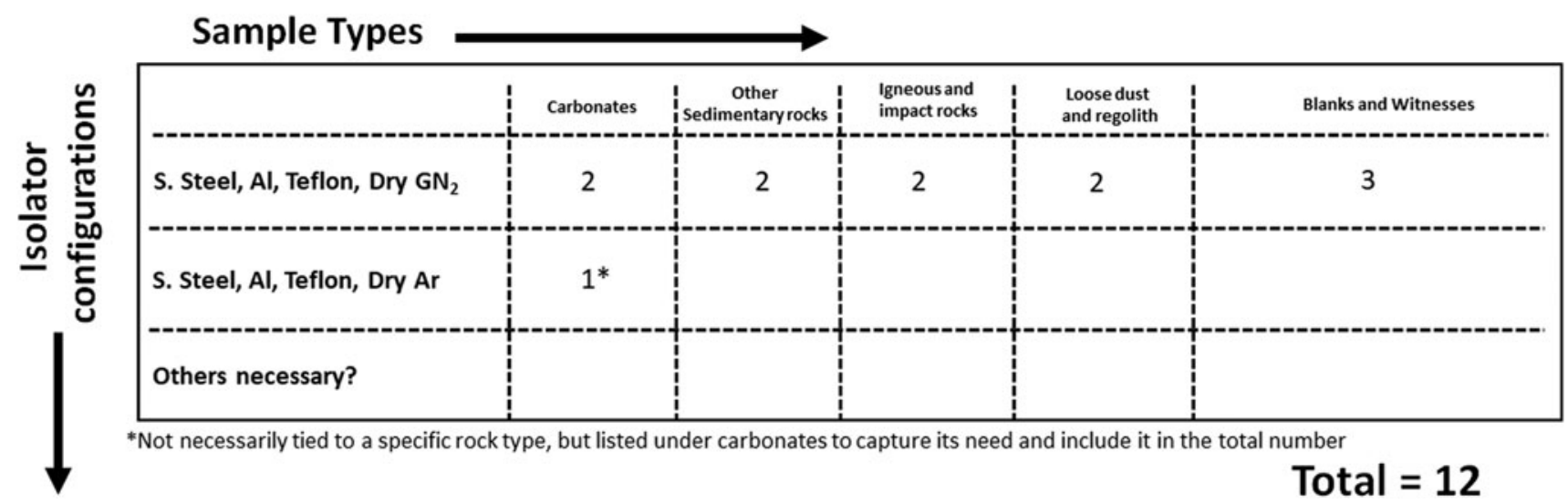

FIG. 8. Matrix of number of sample types vs. isolator configurations for the $\mathrm{SRF}, \mathrm{GN}_{2}=$ gaseous nitrogen.

There is a balance to be found for the number of isolators in the SRF to optimize both schedule and cost. To mitigate risk of work stoppages due to unforeseen problems and/or arduous cleaning processes, we will need to have at least one backup cabinet. The total number of isolators will increase by two for each additional distinct processing environment (Figure 8).

MAJOR FINDING C-11: Assuming that sample processing rates are reasonable and the samples are organized into five sets for cross contamination avoidance purposes, at least twelve pristine isolators are required to perform Basic Characterization on the MSR samples. This total would increase by two for each additional distinct processing environment.

\subsection{Headspace gas collection}

The sample tubes contain atmospheric gases from Mars that would be useful to the scientific community to extract and measure. The removal of the gases needs to be done carefully at the start of the BC process (after dust removal, XCT measurements, magnetometer measurements). Regardless of local environmental perturbations that may occur on the surface of Mars or perturbations triggered once the samples are drilled and briefly exposed to ambient atmosphere during sealing in the tube, puncturing the sample tube seal and extracting the headspace gas would perturb local equilibrium conditions between gas and rock and set in motion volatile exchange processes that would proceed as a function of time. This effectively "starts the clock" on timesensitive measurements. See more discussion on this in the companion MPSG2 document related to time-sensitive measurements (Tosca et al., 2021).

The headspace gas could be extracted from the sample tubes while they are in the STIC or outside of the STIC. It would be advantageous for each sample tube container to have a piercing mechanism that can be connected to a gas manifold system that can extract and measure the gas. After gas extraction, the interior of the STIC, including the sample tube, should be filled with a curation gas (TBD).
The best way to extract and analyse the headspace should be the focus of a future working group (see Section 8.1, Future Work 10).

\subsection{Opening of the sample tubes}

After the gas is extracted, the solid sample would be removed from the sample tube. In the case of the rock cores, it will be critical that the sample inside the tube is minimally disturbed during this process and that primary spatial information is retained, such as laminations or stratigraphic positioning within the tube. In the case of regolith, the sample acquisition process does not retain stratigraphy or orientation (the sample is in effect poured into the tube), so avoiding disturbance for such samples is far less important. However, the specifics of exactly how to open the tubes has not been finalized, and there may be trade-offs between aspects of the opening process and the degree of disturbance of the precious rock cores. It will be a scientific priority that the tubes be opened lengthwise (not dumped out from the top or the bottom of the tube), and that we minimize contamination of the samples with tube material (e.g., metal filings). We note that great care will need to be taken to ensure that vertical orientation of the samples (i.e., the stratigraphic up direction) is preserved and as much care as possible is taken for the spatial relationship information retained. There will need to be future work to establish baseline planning and the associated mechanical systems (see Section 8.1, Future Work 11).

\subsection{Basic Characterization Summary}

A primary purpose of the $\mathrm{BC}$ phase is to provide the information required for a single, crucial decision: How to take the first set of subsamples from original whole rock samples? These subsamples would be used as part of both the PE and SSAP processes, and in both cases, it is essential to understand how each subsample relates to the original asreceived sample. The key objective is to be able to apply data from subsamples to interpretation of the original full sample mass. A key aspect of this, therefore, is to develop a first order understanding of the nature and scale of sample heterogeneity. Note that, because of the way M2020 will acquire the samples (the drill will acquire them directly into 
Pre-BC

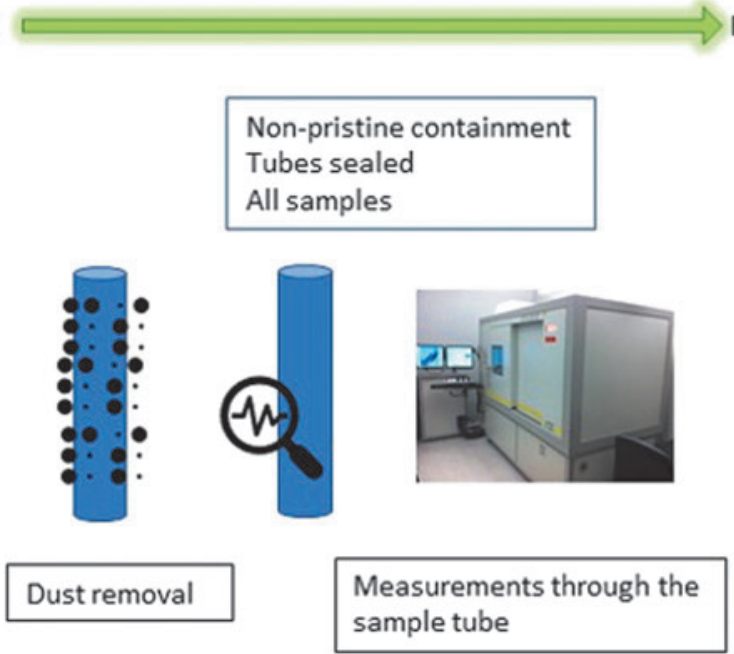

$\mathrm{BC}$

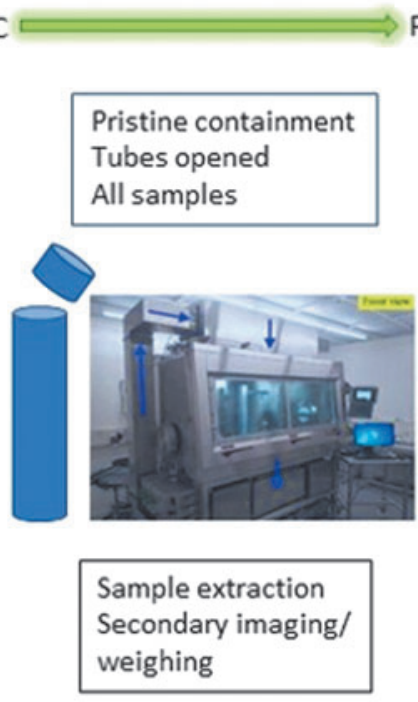

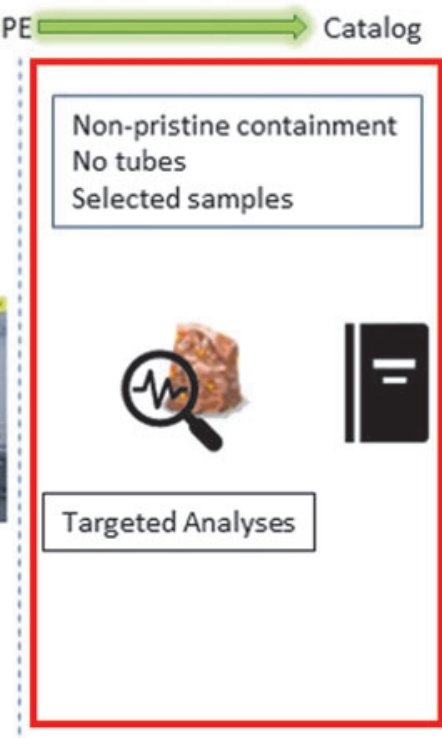

FIG. 9. Proposed sequence of activities within the Pre-Basic Characterization, Basic Characterization, and Preliminary Examination, with the Preliminary Examination stage enclosed within a red box.

the tubes), its instruments will have an opportunity to observe heterogeneity in the outcrop plane, the general area of sample collection, but not in the sample itself. In addition, for reasons of contamination control, during the $\mathrm{BC}$ phase the samples would be confined to pristine isolators, and severe restrictions regarding touching them should be in place. Scientific observations would almost entirely need to be done through windows into the isolators. We recognize only two instruments that are useful in this configuration: a Binocular Stereo Light Microscope and Multispectral Imaging/ Hyperspectral Scanning. In summary, the instrument options for doing whole-rock characterization during the $\mathrm{BC}$ phase is highly limited, and the two instruments named above are considered absolutely essential.

\section{Preliminary Examination (PE)}

The data generated by $\mathrm{BC}$ would be used to design a PE program for each sample. Depending on how significant the differences are between samples, the PE sub-sampling and data collection steps may be significantly different between samples. Note that the PE phase allows for both subsampling and sample preparation, both of which make irreversible changes to the sample. For example, the decision may be made to extract soluble organics from one or more samples (or subsamples). The sample extract could be rendered sterile, either as a direct consequence of the preparation technique (e.g., acid hydrolysis, see Velbel et al., 2021) or possibly through sub-micron filtration, and it could then be transferred outside of biocontainment for analysis. Note that in our usage of the term, PE would happen once, in the SRF, to guide certain critical early decisions, and would not be repeated later. The primary objective of PE would be to provide enough information for the following:

- Principal Investigators (with their supporting teams) to submit relevant and specific proposals for the scientific study of the samples;
- The design of consortium sample studies;

- A sample allocation committee to make informed decisions about the best use of limited, high-value, and irreplaceable sample mass.

Some examples of PE include creating subsamples and performing specific analytical steps deemed necessary to make the above decisions. In contrast to $\mathrm{BC}, \mathrm{PE}$ differs between samples and subsamples depending on what data are required for each sample. These analytical steps may take place in the SRF, elsewhere in biocontainment, or even outside of biocontainment (using either sterilized samples or post-SSAP samples). PE for each sample is expected to last a constrained amount of time, and then the samples and subsamples can be made available for competitive science investigations (see Figure 9).

MAJOR FINDING C-12: More advanced measurements on subsamples, beyond those included in $\mathrm{BC}$, are essential for the allocation of material to the scientific community for investigation, including some measurements that can make irreversible changes to the samples. These types of measurements take place during Preliminary Examination (PE).

\subsection{Envisioned processes and workflows}

After BC is complete on a sample, PE can start. There will be a suite of analytical instrumentation available, depending on the type of information required by the researcher to make an informed decision on the sample they would like to request for their lab-based research within or outside the SRF (see Haltigin et al., 2021). The goal of the $\mathrm{PE}$ stage is to enhance the data collected in Pre-BC and $\mathrm{BC}$, but this has been separated into a distinct stage as not all the samples will be handled in the same way during this stage 
(Figure 9). It was discussed at length that there is a trade-off for obtaining this information. Some instruments will irreversibly change the material. For example, to make a thin section, some material will need to be subsampled, but in doing so, a thin section will be made available for laboratorybased science. The Curation team selected will need to have previous experience with making these types of decisions and will require input from the Campaign Science Group (see Haltigin et al., 2021) and the PIs on the type of sample preparation and data required for their studies.

\subsection{Sample catalog}

One of the key goals of all the Curation activities within the SRF is to prepare a sample catalog for the scientific community to be able to request material that is suitable for their research objectives. The sample catalog should be a living document that contains information from the M2020 Rover (the sample dossier) and from Pre-BC, BC, and PE, and as samples are studied, the sample catalog should be updated to reflect new information collected by the scientific community. When and how the sample catalog is distributed to the scientific community needs to be outlined in a future Rules of Road document.

The way the sample mass is used and subsampled within the SRF is dependent on how full the tubes are when received and planning considerations with regard to how much sample material to hold for the future. After extensive debate within the community, it was determined that the necessary science could be done with the $15 \mathrm{~g}$ rock samples to be returned (and somewhat smaller regolith samples), using the assumption that at least $40 \%$ of the sample mass would be preserved for the future (McLennan et al., 2011; Beaty et al., 2019). The $40 \%$ figure should at some point be discussed, but it needs to be informed by knowledge of the actual sample masses collected, the number of samples actually (or likely) to be sent to Earth, the improvements in instrument efficiency since the $40 \%$ figure was written, and an updated understanding of the time-value of the information in the larger context of planetary science in general (See Section 8.1, Future Work 12).

The sample catalog should be populated with data and information generated during all phases of activity, including data derived from the landed Mars 2020 mission, during sample collection and transport to Earth and reception within the SRF. Data on specific samples and subsamples will also be generated during curation activities carried out within the SRF, including sample safety assessments, timesensitive studies and Pre-BC, BC, and PE phases. The sample catalog will be a living document, meaning that it will be updated regularly as the sample information is collected and processed. There will need to be a "Rules of Road" companion document prepared to outline the details of data distribution and release timing. The sample catalog should provide data for the scientific community to make informed requests for samples for scientific investigations and for the approval of allocations of appropriate samples to satisfy these requests. As material is returned from PI's laboratories, detailed information with regard to the analytical measurements, any sample preparation or processes carried out, and any possible contamination will need to be added into the sample catalog.
For allocation of samples available for use in laboratories outside the SRF, there are two main mechanisms for doing so: 1) Wait until SSAP (Planetary Protection) concludes that the samples are non-hazardous, 2) Render splits of the samples non-hazardous by means of sterilization.

FINDING C-13: The output of the initial sample characterization, and a key function of the curation activities within the Sample Receiving Facility, is to produce a sample catalog that would provide relevant information on the samples' physical and mineralogical/chemical characteristics (derived from the Pre-Basic Characterization, Basic Characterization, and Preliminary Examination investigations), sample safety assessments, time-sensitive studies, and information derived from mission operations to enable allocation of the most appropriate materials to the scientific community.

\subsection{Preliminary examination summary}

Acquiring the MSR samples and transporting them to Earth is expensive, and the quantity of sample material would be constrained relative to the expected scientific demand. For these reasons, we need to minimize the risk that sample material would be wasted. A key strategy to reduce this risk is preliminary examination and the publication of a detailed sample catalog. This will be critical for two reasons:

1. The members of the scientific community need to know which samples to request to optimize their needs, and how much. For example, an investigator who wants to measure an $\mathrm{Rb}-\mathrm{Sr}$ internal isochron needs to have a means of estimating the concentration of both of those elements to determine how much material to request. If they request too much, the excess could be wasted. If they request too little, the investigation may generate ambiguous (or nil) results, which would be even more wasteful.

2. The sample allocation committee will need to know in some detail how the various samples differ from each other to make the best fits between the multiple requests and the sample mass available. Obviously, there will be more than one possible solution to the problem, and our goal will need to be to avoid significantly suboptimized solutions.

The quantity of information obtained with the proposed instrumentation that collectively adds up to PE is something of a gray scale; it is difficult to be definitive that any individual instrument must be present. However, the authors of this report feel very strongly that the scientific opportunity represented by MSR is so important that it is worth substantial effort to optimize the decision-making associated with the sample proposal/allocation process.

The instruments described in this report are, in our judgement, both necessary and sufficient to develop enough understanding of the samples that the sample request proposals written by the sample analysis community, and the associated sample allocation decisions, can be managed effectively. In our collective experience, the job would be best done with 7 complementary instruments for PE (FE-VP-SEM, Confocal 
Raman Spectrometer, Deep UV Fluorescence Microscopy, FTIR, Micro X-ray diffractometer, Energy Dispersive X-ray Fluorescence Spectrometer, and petrographic microscope). This set of instruments would give definitive chemical and textural information (for example, concentrations of elements/ compounds, mineralogy, concentration/speciation of organics, grain size, presence of water and other volatiles, and spatial relationships involving all the above) necessary to classify the samples that make up the collection. These parameters are known to vary systematically among the different types of rocks and regolith known to exist on Mars and expected to be sampled from Jezero Crater. The PE information will also feed into the SSAP process and will be used as input to the selection of sample material for time-sensitive PI-led examinations performed within the SRF. We have debated extensively whether it is possible to prioritize these candidate PE instruments, and we have found that we cannot reach agreement on representing any one of them at a lower level of priority-it is the package of data sets together that provides interpretation breadth and accuracy.

\section{Sample Processing}

There would need to be sample processing infrastructure that is outside of pristine sample biocontainment to prepare samples for $\mathrm{PE}$, analysis within biocontainment, and/or sterilization before samples are allocated. This should be divided into microparticle processing and macrosample processing.

\subsection{Microparticle processing}

The ability to manipulate and sample small particles for the scientific community would be required within the SRF. To prepare small (microscale to mm-scale) samples for the research community often involves the transfer of samples from the collection to analytical substrates. These transfers are accomplished by hand (via tweezers or fine-tipped needles) or by utilizing micromanipulation instruments. Freehand manipulation, especially in biohazard suits, can be difficult due to involuntary hand tremors and due to the triboelectric charge induced by frequent contact between the manipulation tool and the support substrate (Snead et al., 2019). For more information on the instruments recommended for microparticle processing, see Section S-4.1.

\subsection{Macrosample processing}

The ability to manipulate and sample larger pieces of material for sample allocation, thin and thick sections would be required in the SRF. Typically, macrosample processing is "dirty," meaning that it can create dust (which we will want to collect), and therefore should be separate from the microparticle processing area and the PE analytical instrumentation. The curation FG identified the ability to cut samples with a dry wire saw; the ability to prepare thick and thin sections for light microscopy, SEM etc. was identified. We suggest a rock splitter, and there was a brief discussion about tools to be used in both pristine areas and macrosample processing; specifically, concerns were raised about stainless steel for the Highly Siderophile Element (HSE) community. For more information on the instruments recommended for microparticle processing, see Section S-4.2.

\section{Timing and Organizational Considerations}

The Curation activities within the SRF would not be pursued in isolation. Several concurrent activities that would be occurring within the facility should be noted, such as hardware de-integration with engineers, SSAP (Planetary Protection), the Time- and Sterilization-Sensitive measurements, interfacing with the science investigators of competitively selected research within the SRF, and the documentation and data management that will need to occur to produce a sample catalog.

The SRF needs to be adequately staffed, from a team of curation staff that oversee the workflow, to technical staff that operates the instruments, to data facilitation and IT support (see Haltigin et al., 2021). Teams working within the SRF would be under intense pressure and scrutiny from the public, the scientific community, and the media. The SRF could operate 24/7, 5-7 days a week to meet the operational demands, if needed. Instrument technical staff would need to be on hand to operate the instruments, as well as calibrate and tune the instruments as required. Instrument experts should be available on short order to fix or correct any operational issues of the instruments immediately, and to support on-site technical staff as needed. There will need to be sample preparation experts available to support preparing thin sections, thick sections, and coating of samples for the FE-SEM. There needs to be staff dedicated to the cleanliness of the pristine isolators and making them available for the next sample to be processed. There needs to be informatics support staff updating the sample catalog as information is made available.

FINDING C-14: A staffing model for curation activities, including technical support and informatics/ documentation support, should be developed (as part of ongoing Sample Receiving Facility development) to ensure that the Sample Receiving Facility is staffed appropriately to support sample curation activities.

Operating a facility that is simultaneously a cleanroom and a BSL-4 facility is going to place numerous demands that are difficult and expensive to maintain long-term. Once/ if the need for BSL-4 containment has gone away, operations of maintaining the collection can be simpler, less expensive, and more efficient for processing the samples, by moving to a traditional cleanroom-style Curation and/or Storage facility(ies) (Figure 10). It is most likely that this transition would happen within a few years of first receipt of samples, but since we cannot know in advance that the samples will pass their safety assessments, we need to be prepared for the contingency that the SRF is operated indefinitely.

The Curation FG recognizes the risk due to catastrophic loss of having all the samples in one place. The mitigation to this risk is self-evident-divide the collection and hold it in more than one location. This methodology has been used for other precious extraterrestrial samples, for example, NASA curating subsamples of the JAXA Hayabusa and Hayabusa2 samples; NASA storing a sub-set of Apollo samples at a storage facility at White Sands Testing Facility (Calaway et al., 2019). In the case of MSR, it may be impractical to divide the collection during the first two years after receipt of samples - it would increase cost and operational complexity to operate two SRFs. The Curation FG concludes 
On Mars during sample recovery
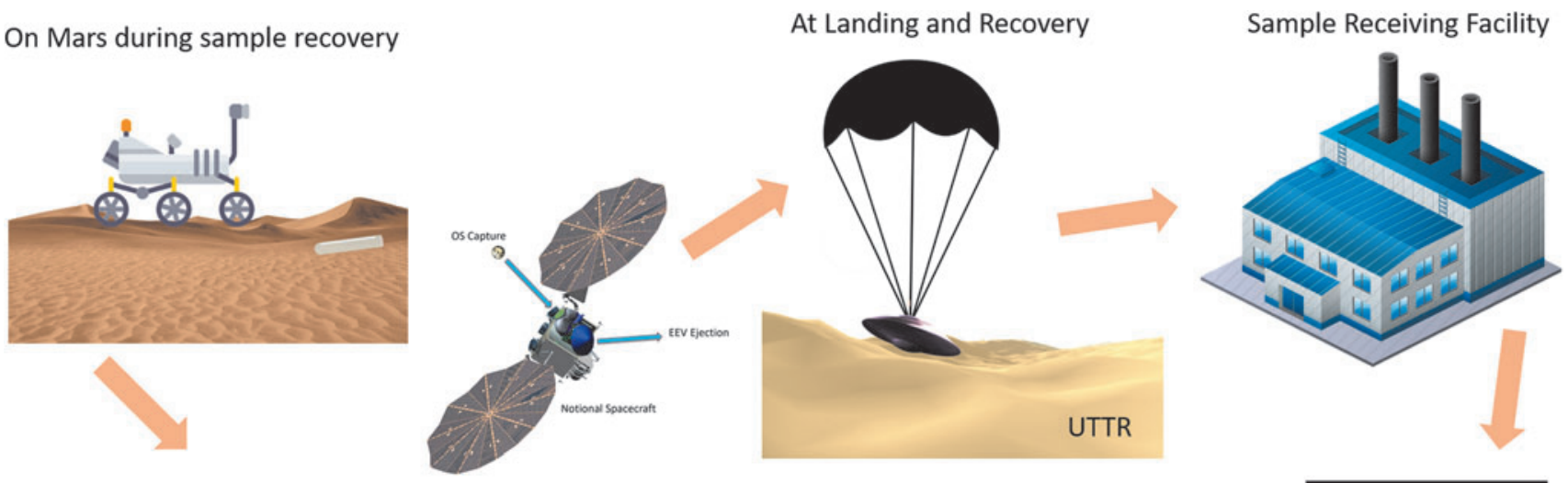

Environmental Monitoring in Space

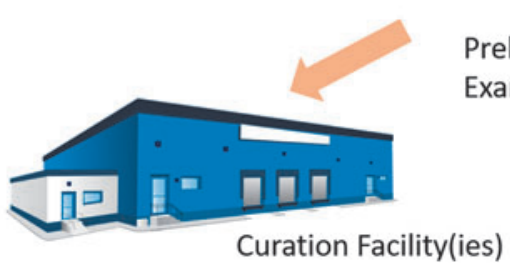

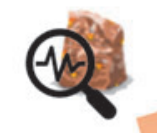

Preliminary Examination

FIG. 10. End-to-end curation schematic. Graphic OS:

that, even though the risk of loss is higher by keeping the samples together in a single SRF, the risk is acceptable because the time-period is relatively short. However, once it is possible to do so, and for the purpose of the long-term safety of the collection, the sample set should be split and housed in more than one separated location.

A model for the division of samples to multiple facilities should be made as soon as possible, and we recommend that this is considered as part of a detailed risk assessment exercise by another group (See Section 8.1, Future Work 15). It will be necessary for this group to consider the mitigation of risk of loss or damage to samples as well as potential benefits or risks to curatorial operations sample accessibility if samples are curated and/or stored across multiple sites.

FINDING C-15: To reduce the risk of catastrophic loss of samples curated in a single facility up to, and including, decadal timescales, the sample collection should be split-once it is possible to do so-and housed in more than one location for the purpose of maximizing the long-term safety of the collection.

\section{Future Work and Conclusions}

\subsection{Future work}

As part of the Curation FG discussions, there were several issues that were not resolved, largely due to time constraints and the need for greater input from outside experts, or where more data are needed. In this section, we outline some of the main points that should be resolved in future work by subject matter experts.
Future Work 1. Electrostatic and humidity considerations within the SRF (Section 2): This was not discussed as part of this discussion group but was noted in review. We recommend a future group to consider these parameters in relation to sample handling.

Future Work 2. Detailed Contamination Control (CC) measurement and verification protocols for sample handling tools, containers, and other equipment (such as gloveboxes, isolation chambers, and desiccators as needed) (Section 2.4.1.): For example, we would need to check regularly for particle counts within clean lab spaces, monitor the trace gases in the curation-grade gases used in the processing cabinets, as well as check the isotopic compositions of these gases and of the UPW. Microbial contamination in the UPW, air filter systems, labs, and within the gloveboxes needs to be monitored. Witness plates to monitor for organic and inorganic contamination should be set out in spaces where pristine samples are handled.

Future Work 3. Determining the best curation gas needed for the isolators (Section 2.4.2.): This was discussed in the Curation FG, and although we concluded nitrogen gas makes the most sense for most cases where detecting martian $\mathrm{N}$ isotopes is not central, there was differing conclusions in the Time-Sensitive FG, so we suggest that this be the focus of a future working team to take in considerations for both curation needs and time-sensitive studies.

Future Work 4. Define the controlled list of materials allowed in the pristine isolator (Section 2.4.5.): It would be crucial to minimize the number of materials that come into direct contact with samples to minimize contamination but still be able to process the samples relatively easily. The working list, as used by JSC in prior missions, should be considered as a starting point for planning and discussion. 
Future Work 5. Determine the best way to remove the dust from the outer surface of the sample tubes and OS interior hardware surfaces, without disturbing the samples within the tubes (Section 3.2.1.): A quick way of removing the dust that does not negatively impact the samples within the tubes and that minimally changes the dust sample on the outside of the tubes should be developed.

Future Work 6. Conduct advanced leak testing of the sample tubes (Section 3.2.2.): We need to know the leak rate of the sample tubes to better understand the sealing capacity of the sample tubes prior to landing.

Future Work 7. Determine methods to check the integrity of the seals, including the PCV, SCV, and sample tubes on the returned spacecraft (Section 3.2.2.): We would like to be able to measure the leak rates on the sample tubes as quickly as possible after the OS is opened. The best samples will be the ones for which the seals on the sample tubes have the lowest leak rate, and that needs to be a documented part of the sample catalog. Unfortunately, we have not been able to identify a way to make this measurement without getting access to both sides of the seal (e.g., similar to how a He leak detector works). It may not be possible to make this measurement accurately while the natural sample gas is still inside the tube. It may be necessary to extract the gas, then evaluate the quality of the seal based on the gas composition.

Future Work 8. Design a Sample Tube Isolation Chamber (STIC) (Section 3.2.3.): The sample tubes were not designed for long-term storage, and integrity of the seals could be compromised. However, the interaction between Earth atmospheric gases and the gases that are in the sealed tube should be minimized. We propose a concept of a device that could house the sample tubes once the dust has been removed from the exterior of the sample tubes. The STIC may also need to be designed to enable tube seal leak checking and headspace gas extraction. More work needs to be done on this conceptual design to determine, for example, what materials would be best for this and how it interacts with the sample tube for gas extraction.

Future Work 9. Effects of XCT on organic/microbial specimens similar to what we expect to receive from Mars and determination of the number of XCTs required for the SRF (Section 3.4): Given that this technique would be used as part of Pre-BC and could be applied to entire sample tubes, substantial research efforts to evaluate the effects of XCT on samples is warranted and should be supported in the coming years. Lab XCT instruments are reliable and do not typically exhibit a lot of downtime, so only one instrument may be required for the SRF, but a future group tasked with optimizing budget and schedule should evaluate the need for one or more XCT instruments in the SRF.

Future Work 10. Determining the best way to extract the headspace gas from the sample tubes (Section 4.3): The sample tubes contain atmospheric gases from Mars and gases evolved from the sample itself that will be useful to the scientific community to extract and measure. The removal of the gases could be done carefully at the end of the Pre-BC process (after dust removal, XCT measurements, magnetometer measurements) as the removal of the atmo- spheric gases will "start the clock" on time-sensitive measurements on the samples (Tosca et al., 2021). The headspace gas must be extracted in a controlled manner that minimizes sample loss, fractionation, and terrestrial contamination, at best via controlled valves into separate gas containers for immediate mass spectrometric or nondispersive IR spectroscopic measurements (to verify martian or terrestrial composition) and to inform future allocation and sample requests.

Future Work 11. Determine a process to open the sample tubes (Section 4.4): A way to open the sample tubes, without disturbing the interior samples and while retaining any stratigraphic information and fine-scale features, should be developed.

Future Work 12. More discussion and thought on the $\mathbf{4 0 \%}$ of material retained for the future (Section 5.2): The $40 \%$ figure should, at some point, be challenged, but it needs to be informed by knowledge of the actual type (solid core, regolith, dust) and mass of samples collected, the number of samples actually (or likely) to be sent to Earth, the improvements in instrument efficiency since the $40 \%$ figure was written, and an updated understanding of the time-value of the information in the larger context of planetary science in general.

Future Work 13. Deep Ultraviolet Fluorescence Spectroscopy instrument development (Section 5.5): Commercially available instruments do not integrate the wavelengths in a manner that is effective for multiwavelength analysis of a geologic sample where both organics and mineral analysis over multiple scales is necessary.

Future Work 14. Motorized micromanipulators for use in the Isolators (Section 6.1.1.): Motorized micromanipulators are used to manipulate small objects or specimens under a microscope. A benefit to using motorized micromanipulators is the precision movement on a variety of axes that enables absolute manipulation far superior to traditional manual manipulation.

Future Work 15. Risk assessment for the samples (Section 7): There was discussion about all the samples being in one place, even if it is for several years during SRF operations, and there is a risk to having all the samples in once place should a catastrophic event occur. It will be necessary for this group to consider the mitigation of risk of loss or damage to samples as well as potential benefits or risks to curatorial operations sample accessibility if samples are curated and/or stored across multiple sites.

RECOMMENDATION: The outstanding questions related to analytical instruments that could support Mars Sample Return goals should be communicated to the greater scientific community.

\subsection{Conclusions}

The Mars Sample Return Science Planning Group-2 Curation FG has evaluated the science objectives set forth in the iMOST report (Beaty et al., 2019) and has determined that there should be three stages of characterization of the samples split into Pre-BC, BC, and PE. For Pre-BC, a XCT 
and magnetometer is required. For BC, an analytical balance, a binocular light microscope, multispectral imaging, and hyperspectral scanning are required.

For Preliminary Examination, a series of analytical instruments should be available in the Sample Receiving Facility to prepare the sample catalog. They are a Variable Pressure Scanning Electron Microscope, a Field-Emission Scanning Electron Microscope, a confocal Raman Spectrometer, a Deep UV Fluorescence Spectrometer, Fourier Transform Infrared Spectrometer, Micro X-ray Diffractometer, and an X-ray Fluorescence Spectrometer with supporting petrographic and stereo microscopes. These instruments would be used when required for a measurement that supports the building of the sample catalog and therefore would support the science, as well as the allocation process, well into the future.

\section{Acknowledgments}

We would like to thank the entire MSPG-2 committee for their input and discussions during our bi-weekly meetings, and especially Gerhard Kminek, Tim Haltigin, Nicholas Tosca, and Michael Velbel for their input on this document. We especially acknowledge the subject matter experts Professor Ben Weiss (MIT) and Dr. Rohit Bhartia (Photon Systems Inc) for their important input on magnetometers and Deep UV Fluorescence, respectively. We also thank Dr. Paul Northrup and Dr. Mark Rivers for their input on synchrotron radiation techniques and Dr. Juliane Gross (Rutgers University/NASA Johnson Space Center) and Dr. Paul Lucey (University of Hawaii, Manoa) for their discussions on Multispectral Imaging and Hyperspectral Scanning and as well Dr. Ryan Ziegler and Scott Eckley (NASA, Johnson Space Center) for discussions and input on X-ray Computed Tomography. We also appreciate comments from an SRF implementation team coordinated by Richard Mattingly.

The decision to implement Mars Sample Return will not be finalized until NASA's completion of the National Environmental Policy Act (NEPA) process. This document is being made available for planning and information purposes only.

\section{Disclosure Statement}

No competing interests.

\section{Funding Information}

A portion of this work was funded by the National Aeronautics and Space Administration (NASA) and the European Space Agency (ESA).

A portion of this work was carried out at the Jet Propulsion Laboratory, California Institute of Technology, under a contract with the National Aeronautics and Space Administration (80NM0018D0004).

This work has partly (H. B.) been carried out within the framework of the NCCR PlanetS supported by the Swiss National Science Foundation. M.A.V's participation in MSPG2 was supported in part by a sabbatical leave-ofabsence from Michigan State University. M.-P.Z. was supported by projects PID2019-104205GB-C21 of Ministry of Science and Innovation and MDM-2017-0737 Unidad de Excelencia 'Maria de Maeztu'- Centro de Astrobiología (CSIC-INTA) (Spain).

\section{References}

Baker MJ, Trevisan J, Bassan P, et al. (2014) Using Fourier transform IR spectroscopy to analyze biological materials. Nat Protoc 9:1771-91.

Beaty DW, Grady MM, McSween HY, et al. (2019) The potential science and engineering value of samples delivered to Earth by Mars sample return. Meteorit Planet Sci 54:S3S152.

Calaway MJ, Allton JH, McCubbin FM, et al. (2019) The rise of astromaterials curation: NASA's 50 years of preserving pristine samples from the Solar System [abstract 2132]. In 50th Lunar and Planetary Science Conference, Lunar and Planetary Institute, Houston.

Calaway MJ, Rodriguez MC, Alton JH, et al. (2009) Decontaminating solar wind samples with the Genesis ultra-pure water megasonic wafer spin cleaner [abstract 1183]. In 40th Lunar and Planetary Science Conference, Lunar and Planetary Institute, Houston.

Day JMD, Maria-Benavides J, McCubbin FM, et al. (2018) The potential for metal contamination during Apollo lunar sample curation. Meteorit Planet Sci 53:1283-1291.

Grady MM, Summons RE, Swindle T, et al. (2022) The scientific importance of returning airfall dust as part of Mars Sample Return. Astrobiology 22(S1):S-176-S-185.

Haltigin T, Hauber E, Kminek G, et al. (2022) Rationale and proposed design for a Mars Sample Return (MSR) science program. Astrobiology 22(S1):S-27-S-56.

Harrington AD, Calaway MJ, Regberg AB, et al. (2018) The importance of contamination knowledge in curation-insights into Mars Sample Return [abstract 2083]. In 49th Lunar and Planetary Science Conference, Lunar and Planetary Institute, Houston.

Libourel G, Ganino C, Delbo M, et al. (2021) Network of thermal cracks in meteorites due to temperature variations: new experimental evidence and implications for asteroid surfaces. Monthly Notices of the Royal Astronomical Society 500:1905-1920.

McCubbin FM, Herd CDK, Yada T, et al. (2019) Advanced curation of astromaterials for planetary science. Space Sci Rev 215, doi:10.1007/s11214-019-0615-9.

McLennan SM, Sephton MA and Allen C (2012) Planning for Mars Returned Sample science: Final report of the MSR Endto-End International Science Analysis Group (E2E-iSAG). Astrobiology 12:175-230.

Moeller RC, Jandura L, Rosette K, et al. (2021) The Sampling and Caching Subsystem (SCS) for the scientific exploration of Jezero Crater by the Mars 2020 Perseverance Rover. Space Science Reviews 217:1-43.

Russell S, Smith CL, Hutzler A, et al. (2019) EURO-CARES A European sample curation facility for sample return missions. In 2019 IEEE Aerospace Conference, Big Sky.

Sears DWG, Sears H, Ebel DS, et al. (2016) X-ray computed tomography imaging: A not-so-nondestructive technique. Meteorit Planet Sci 51:833-838.

Snead CJ, Cowden TR and McCubbin FM (2019) Development of low-cost micromanipulation systems for small extraterrestrial samples. In Annual Meeting of the Meteoritical Society, Sapporo.

Swindle TD, Atreya S, Busemann H, et al. (2022) Scientific value of including an atmospheric sample as part of Mars Sample Return (MSR). Astrobiology 22(S1):S-165-S-175.

Tosca NJ, Agee CB, Cockell CS, et al. (2022) Time-sensitive aspects of Mars Sample Return (MSR) Science. Astrobiology 22(S1):S-81-S-111. 
Younse P, Strahle JW, Dolci M, et al. (2018) An orbiting sample capture and orientation system architecture for potential Mars Sample Return. In 2018 IEEE Aerospace Conference, Big Sky.

Zeigler RA, Hanna R, Edey D, et al. (2021) Using X-ray Computed Tomography to image Apollo Drive Tube 73002. In 52nd Lunar and Planetary Science Conference, Lunar and Planetary Institute, Houston.

For further information about MSPG2, please contact Michael Meyer (Michael.a.meyer@nasa.gov),

Gerhard Kminek (Gerhard.kminek@esa.int),

David Beaty (dwbeaty@jpl.nasa.gov),

or Brandi Carrier (bcarrier@jpl.nasa.gov).

For further information on the technical content of this report, contact any of the above or Kimberly Tait (ktait@rom.on.ca), Francis McCubbin (francis.m.mccubbin@nasa.gov), or Caroline Smith (caroline.smith@nhm.ac.uk).

$\begin{aligned} & \text { Acronyms Used } \\ \mathrm{BC}= & \text { Basic Characterization } \\ \mathrm{BSL}-4= & \text { Biosafety Level } 4 \\ \mathrm{COSPAR}= & \text { Committee on Space Research } \\ \mathrm{CSG}= & \text { Campaign Science Group } \\ \mathrm{EEV} / \mathrm{EES}= & \text { Earth Entry Vehicle/Earth Entry } \\ & \text { System; a subsystem of the Earth } \\ & \text { Return Orbiter spacecraft } \\ \mathrm{ESA}= & \text { European Space Agency } \\ \mathrm{FE}= & \text { Field-emission } \\ \mathrm{FG}= & \text { Focus Group } \\ \mathrm{FTIR}= & \text { Fourier transform infrared } \\ \mathrm{HSE}= & \text { Highly Siderophile Element }\end{aligned}$

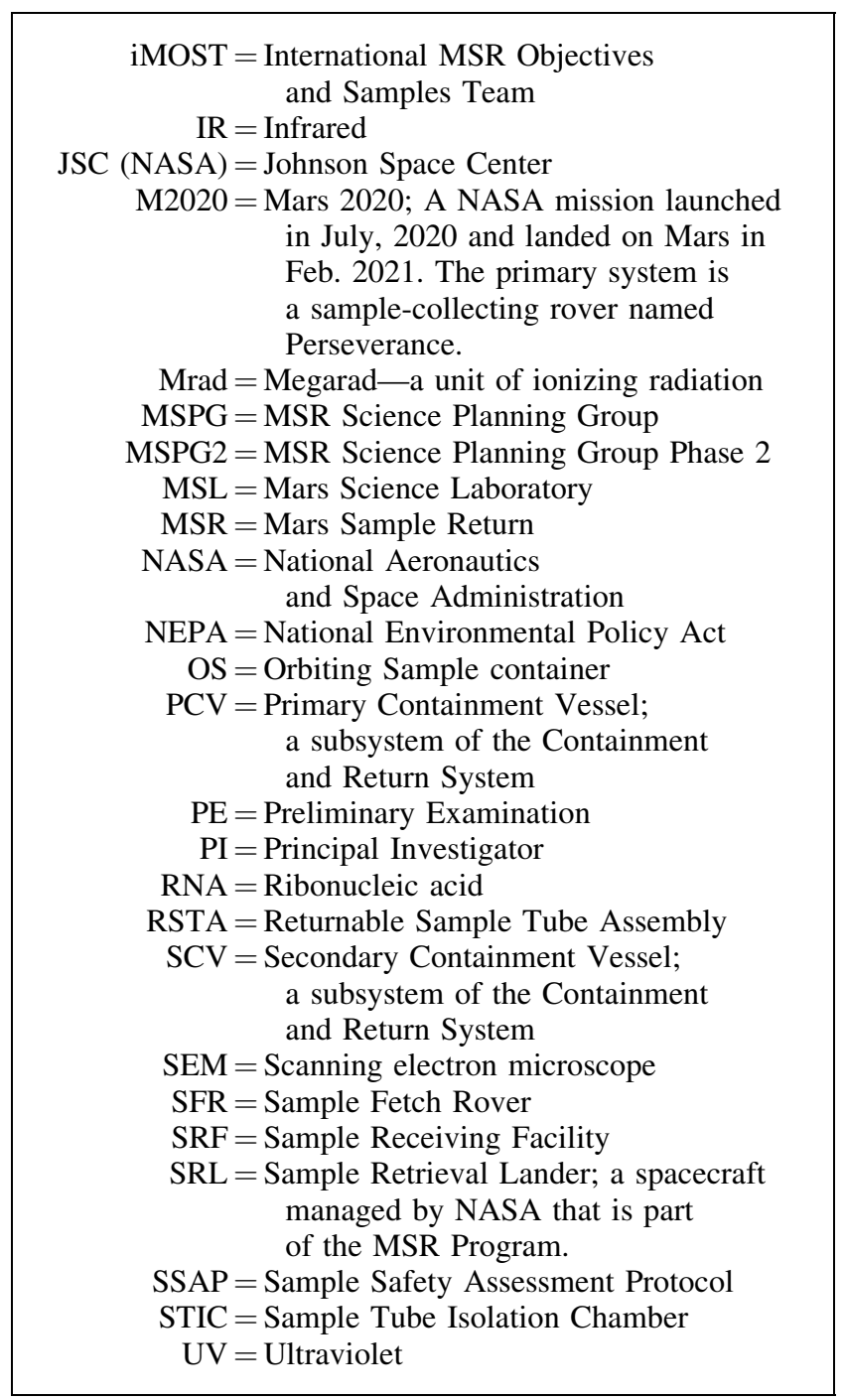

